\title{
Modelling the tonotopic map using a two-dimensional array of neural oscillators
}

1 Dipayan Biswas ${ }^{1}$, V. Srinivasa Chakravarthy ${ }^{*}$, Asit Tarsode ${ }^{12}$

$2 \quad{ }^{1}$ Laboratory for Computational Neuroscience, Department of Biotechnology, Bhupat and Jyoti Mehta

3 School of Biosciences, Indian Institute of Technology Madras, Chennai, India

$4 \quad{ }^{2}$ Department of Mechanical Engineering, Indian Institute of Technology Madras, Chennai, India

5 * Correspondence:

6 Corresponding Author

7 schakra@ee.iitm.ac.in

8 Keywords: self-organizing map1, tonotopy2, modified power coupling, interference4,

9 resonances, Hopf oscillator6, entrainment 7 , synchronizations.

10 Abstract

11 We present a model of a tonotopic map known as the Oscillatory Tonotopic Self-Organizing Map 12 (OTSOM). It is a 2-dimensional, self-organizing array of Hopf oscillators, capable of performing a 13 Fourier-like decomposition of the input signal. While the rows in the map encode the input phase, the 14 columns encode frequency. Although Hopf oscillators exhibit resonance to a sinusoidal signal when 15 there is a frequency match, there is no obvious way to also achieve phase tuning. We propose a simple 16 method by which a pair of Hopf oscillators, unilaterally coupled through a coupling scheme termed as modified power coupling, can exhibit tuning to the phase offset of sinusoidal forcing input. The training of OTSOM is performed in 2 stages: while the frequency tuning is adapted in stage 1, phase tuning is adapted in stage 2. Earlier tonotopic map models have modeled frequency as an abstract parameter unconnected to any oscillation. By contrast, in OTSOM, frequency tuning emerges as a natural outcome of an underlying resonant process. The OTSOM model can be regarded as an approximation of the tonotopic map found in the primary auditory cortices of mammals, particularly exemplified in the studies of echolocating bats.

\section{Introduction}

The discovery of cortical brain maps in mammalian brains is perhaps one of the first milestones in our understanding of how the brain generates representations of the world. Visual research had discovered a rich hierarchy of maps of various sub-modalities of vision (orientation, curvature, color, and even complex objects) in various visual cortical areas extended over the occipital, parietal, and temporal lobes (Hadjikhani et al., 1998; Hubel \& Wiesel, 1959; Wandell et al., 2007; Yue et al., 2020). A similar network of maps of somatotopy was found in the somatosensory areas of the postcentral gyrus, and posterior parietal cortex (Penfield, 1937). These studies have placed on a firm foundation the understanding that sensory information in the brain is often laid out in the form of a system of 
33 topographic maps. However, efforts to establish a similar map structure underlying auditory processing

34 - popularly referred as tonotopic maps, - are met with considerable challenges.

35 Tonotopy begins in the inner ear, in the hair cells laid out along the length of the basilar membrane inside the cochlea (Bekesy, 1949). Parts of the basilar membrane respond to different frequencies, with the tuning frequency increasing in the apex to the base direction (Ruggero, 1992). Thus, there is a wellestablished tonotopy in the cochlea, sometimes also referred to as cochleotopy. Beyond the cochlea, there is a hierarchy of areas along the auditory pathway (Clopton et al., 1974; Ehret \& Romand, 1996; Palmer \& Rees, 2010). Although there is a general agreement that what is mapped in tonotopic maps is the frequencies, other auditory parameters like sound intensity, tuning bandwidth are also explored (Boynton et al., 2015; Schreiner \& Sutter, 1992).

Earliest studies on tonotopy focused on frequency tuning, treating it as one of the primary features if not the sole defining feature of auditory response. Merzenich et al., 2018 found a systematic representation of cochlea within the primary auditory cortex of cats. It was observed that frequency bands of the input stimuli are mapped onto rectilinear strips in the auditory cortex. Similar observations were made in the auditory cortex of the grey squirrel (Michael M. Merzenich et al., 1976). Investigations of the auditory cortex in owl monkeys have discovered a central area with orderly mapping of audible frequencies, circumscribed by areas where neurons show more complex responses than frequency tuning (Imig \& Adrian, 1977). However, the exact nature of the complex responses was not elaborated in the last study.

A hierarchically organized network of areas with complex information processing properties was discovered subsequently in the auditory cortex of the bat (N. Suga, 1990). Contrary to popular belief, bats are not visually blind, though the extent of visual capacity varies with different subspecies of bats. But bats predominantly depend on echolocation to navigate through the spatial world. Bats emit ultrasound pulses in the frequency range of tens of kilo Hertz, and interpret the spatial world from the echoes returned by the environment. Whereas the delay between the emitted and the received pulse reveals distance, doppler shift in the echo reveals the relative velocity between the echolocating bat Suga, and others had revealed that these complex auditory functions of the bat are subserved by a well-

61 developed auditory system (Bates et al., 2011; Novick \& Vaisnys, 1964; Simmons, 2012; N. Suga, 1990; Nobuo Suga et al., 1997). 
63 Studies by Nobuo Suga and colleagues with the mustached bat had described an elaborate network of 64 auditory cortical areas with an intrinsic hierarchy not very different from that of the primate visual 65 system (Hubel \& Wiesel, 1959). The mustached bat emits composite pulses that have an initial Constant Frequency (CF) section terminated by a Frequency Modulated (FM) section. There is a cortical region in which neurons respond only to certain combinations of frequencies and amplitudes of echoes. There is a region where neurons respond only to frequency differences between the emitted pulse and its echoes, probably encoding Doppler shift. In another region there are neurons that respond to the time delay between the emitted pulse and the echo, perhaps encoding the distance to the target. The gains obtained from the study of the bat's auditory system are not yet fully exploited in unravelling the auditory architecture of the brains of higher mammals and humans.

In the domain of computational modelling, one of the earliest tonotopic map models used a SelfOrganizing Map (SOM) model to model the auditory cortex of mustached bat (Ritter et al., 1992). The model adopted a simplistic view of the organization of the bat's auditory cortex - that the input frequencies are mapped along a rectilinear strip of the cortex - and shows how such a mapping can be realized using a rectangular SOM model. A key limitation of the model is the representation of frequency as an explicit scalar variable and not as an implicit temporal property of an ongoing oscillation. The SOM approach to modelling tonotopy was extended to construct a model of a "phonetic typewriter" (Kohonen, 1988). Palakal et al., 1995 presented a tonotopic map model also based on the SOM approach, describing neural tuning to both frequency and delay. Here too frequency and delay are explicitly represented as scalar variables, and not as implicit temporal properties of a signal.

Models tend to make simplifying assumptions of the processes they aim to model. But it is rather unnatural to model frequency as simply a number without explicitly modelling the oscillation that the frequency refers to. A tonotopic map is primarily a response to tones which are oscillations. Oscillatory activities are found at all levels in the auditory pathway, from cochlea to inferior colliculus to higher auditory cortical areas. There is a considerable body of literature that examines the application of nonlinear oscillators to describe responses of the auditory system at various levels to periodic stimulus(Frank Julicher et al., 2001; Fredrickson-hemsing et al., 2012; Kim \& Large, 2015; Laudanski et al., 2010; Meddis \& Lowel P. O’Mard, 2006; Víctor M. Eguíluz \& Ospeck, 2000). However, these are single-unit models of oscillation and not map models.

92 From the aforementioned quick review of auditory response models, we understand that there are

93 tonotopic map models that do not explicitly model the underlying oscillation, and there are oscillatory 94 models at single unit level that are not extended to map models. Thus, the challenge of constructing a 
tonotopic map model of nonlinear oscillators is still unrealized, which becomes the motivation of the present work.

97 We present a tonotopic map model consisting of a 2-dimensional array of nonlinear oscillators. 98 Specifically, we choose the Hopf oscillators since these oscillators have been extensively used to model 99 auditory responses (Farokhniaee et al., 2020; Frank Julicher et al., 2001; Fredrickson-hemsing et al., 100 2012; Kim \& Large, 2015; Large et al., 2010). In the following methods section, we have presented 101 the dynamics of the OTSOM model along with the dynamical analysis of a single unit of the OTSOM 102 model and the modified power coupling strategy along with the modified Hebbian learning rule to train

103 it. The dynamical analysis of the two stages to train the characterizing frequencies and the phases of the model is presented thereafter. The numerical analysis from the unit level to the network level is presented in the subsequent results section.

\section{Methods}

108 The conventional self-organizing maps are known for their special characteristic of organizing 109 internally represented features on a spatial scale. i.e., the neurons representing similar abstract features 110 in the input data organize themselves spatially close to each other through competitive learning 111 (Kohonen, 1998). Typically, SOM models perform dimensionality reduction of a high dimensional 112 input vector by projecting it onto a low dimensional spatial map space. In other words, the input data 113 points located nearby in the $N$-dimensional Euclidean space get mapped onto nearby neurons in the 114 map. The map space can be maximum up to 3 dimensional as it is not easily visualized beyond 3 115 dimensions. Also, brain maps are typically 2-dimensional, referring typically to cortical sheets of 116 neurons, or mildly 3-dimensional, if the small cortical thickness is included.

117 The objective of our modelling study is to propose a dynamical self-organizing map model which can 118 organize the features of complex sinusoidal signals on a 2-dimensional grid of nonlinear oscillators. 119 Signals of any duration have a static representation in Fourier space or frequency domain. The proposed 120 model is capable of organizing features of complex sinusoidal signals such as frequency and phase 121 offset, in terms of the parameters of intrinsic dynamics of single neural oscillator and the connectivity 122 parameters during the training phase. During testing, the trained map model can represent the features 123 of any composite signal with multiple frequency components.

\section{Oscillatory Tonotopic Self Organizing Map (OTSOM):}


125 The Oscillatory Tonotopic Self Organizing Map (OTSOM) model consists of a 2D array of Hopf 126 oscillators (Strogatz, 1994) - the "Cortical Array of Oscillators" (CAO) - operating at supercritical

127 Hopf regime as described in fig. 1. A single isolated oscillator located apart from the CAO is interpreted 128 as a subcortical oscillator, and labeled as Subcortical Reference Oscillator (SRO) since it serves as a 129 reference for the phases of the cortical oscillators. Although there are no lateral interactions between 130 the oscillators in CAO, the SRO projects unilateral, trainable connections to all the oscillators in CAO.

131 Thus, each oscillator in CAO receives two inputs: 1) from the SRO (termed as $I_{r}$ ) and, 2) from the 132 external input (termed as $I_{e}$ ) (fig. 1). The connections from the external input to the CAO oscillators 133 have uniform fixed weights.

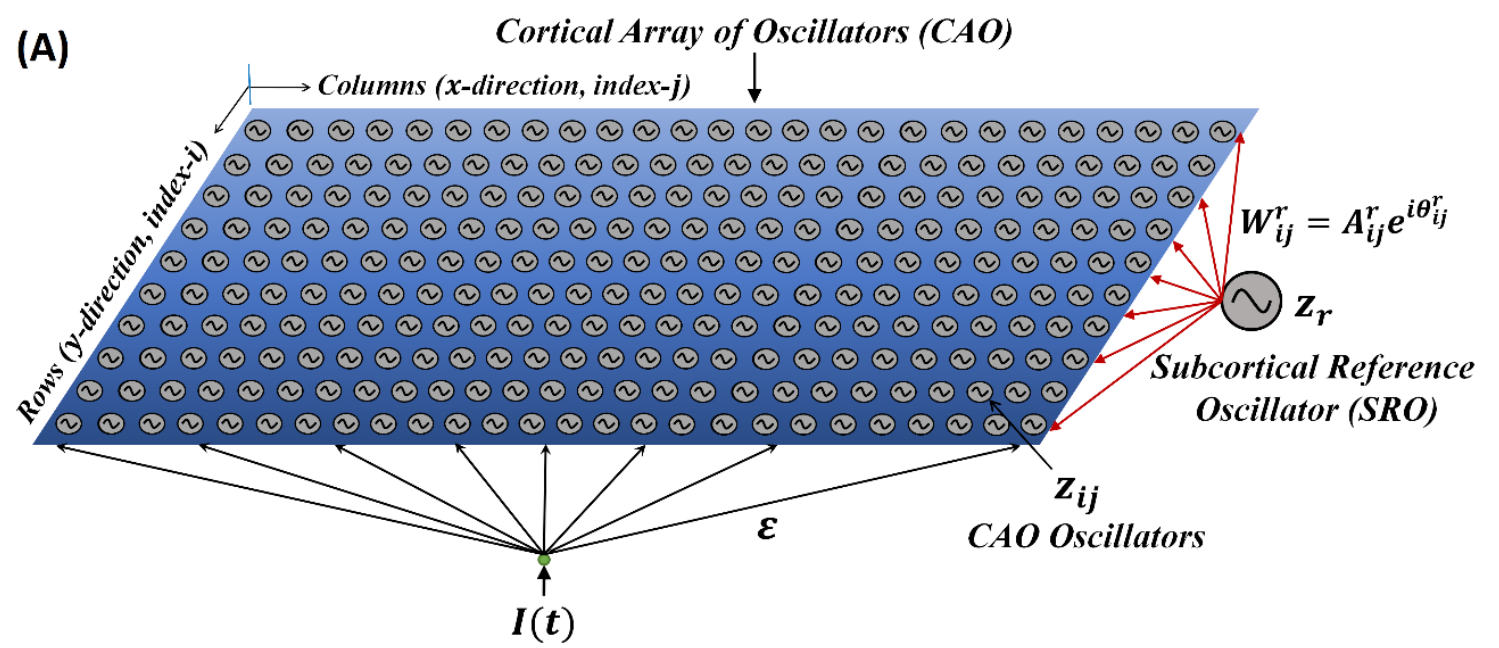

(B)

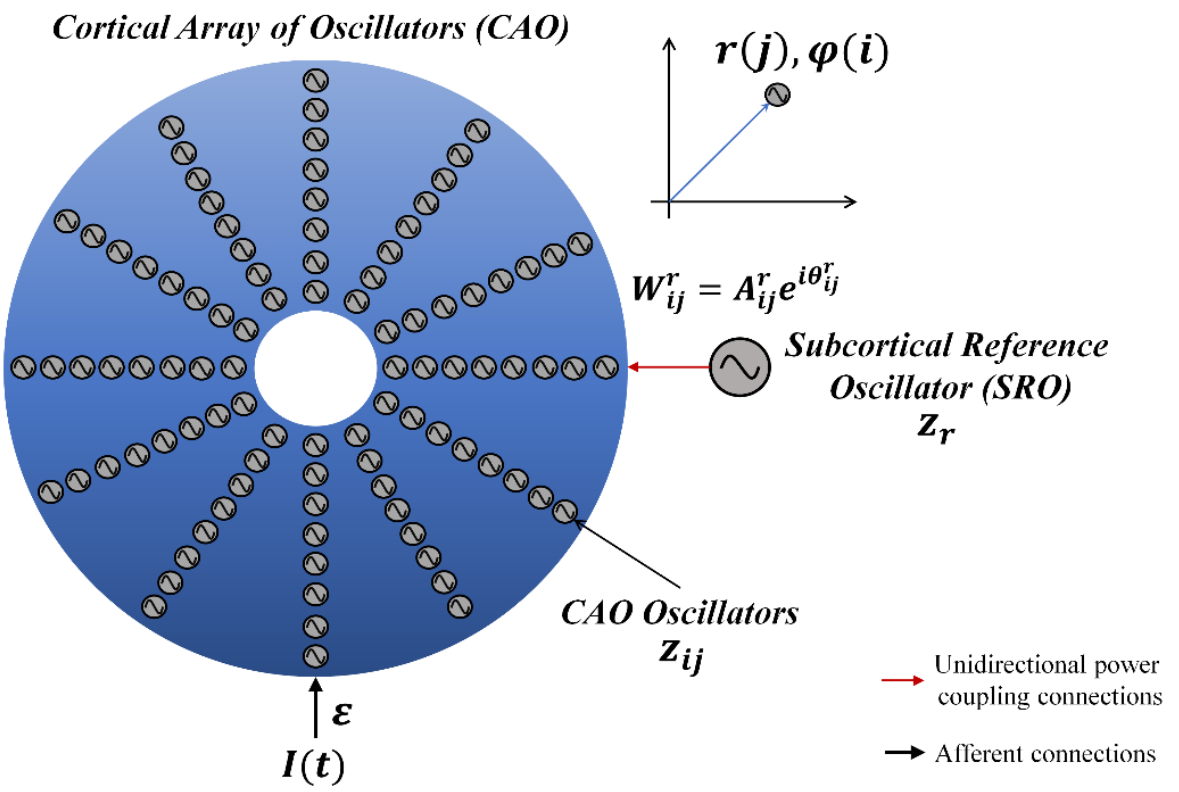

135 Figure 1: The network architecture of Oscillatory Tonotopic Self Organizing Map (OTSOM). The 136 model principally contains two types of oscillators: an array of cortical oscillators (CAO) and a 
137 Subcortical Reference Oscillator (SRO). The SRO projects unilateral, power-coupling connections to 138 the CAO oscillators. The external input perturbs the oscillators in CAO through uniform afferent 139 connections. The CAO oscillators can be visualized to be organized either in a 2D array (A) or in 140 concentric circular array (B). The radial and the angular position of a CAO oscillator in the concentric 141 circular array is same as its position along $\mathrm{x}$ and $\mathrm{y}$ axis respectively in the 2D array organization of the 142 cortical array.

Before going into more details of the dynamics of the OTSOM model let us first understand 144 the dynamics of the constituting components of the OTSOM model. As mentioned before, the CAO 145 oscillators and the SRO are Hopf oscillators. A single Hopf oscillator can be represented in Cartesian 146 (eqns. 1a, b) and polar coordinates (eqns. 2a, b) respectively as follows:

$$
\dot{x}=\left(\mu-\beta_{1}\left(x^{2}+y^{2}\right)\right) x-\omega y
$$

where, $(x, y)$ are cartesian coordinate variables and $(r, \emptyset)$ are polar coordinate variables; $\omega$ defines the angular velocity or the natural frequency of the oscillator. The parameters $\mu$ and $\beta_{1}$ determine the dynamic regime of the Hopf oscillator: for $\mu=0, \beta_{1}>0$ it operates in critical Hopf regime; for $\mu>$ $0, \beta_{1}>0$ it operates in supercritical Hopf regime and when $\mu=0, \beta_{1}=0$ it is a simple harmonic oscillator (Kim \& Large, 2015).

156 Combining $\mathrm{x}$ and $\mathrm{y}$ of eqn. (1) into a complex number, $z=x+i y$, Hopf oscillator dynamics can simply be represented on complex plane elegantly as:

$$
\dot{z}=\left(\mu-\beta_{1}|z|^{2}+i \omega\right) z
$$

where, $i=\sqrt{-1}$. A pair of coupled Hopf oscillators can principally exhibit two types of dynamical phenomena: synchronization and entrainment. 
163 their intrinsic oscillation frequencies if they maintain any of the following phase relationships constant,

$164 \emptyset_{1}-\emptyset_{2}, m \emptyset_{1}-n \emptyset_{2}, \frac{\emptyset_{1}}{\omega_{1}}-\frac{\emptyset_{2}}{\omega_{2}} ; m$ and $n$ are natural numbers. Whereas entrainment is the dynamical

165 characteristic of an oscillator whilst the frequency of oscillation of the oscillator gradually changes

166 from its natural frequency of oscillation to a new value when the oscillator is either coupled with

167 another oscillator or perturbed by an external oscillatory input of a different frequency. Real valued

168 symmetric coupling yields in phase $\left(0^{\circ}\right)$ oscillation for positive coupling, and out of phase $\left(180^{\circ}\right)$

169 oscillation for negative coupling, between two isochronous oscillators, whereas the same pair can

170 phase-lock at any arbitrary phase difference if coupled through 'complex coupling' strategy (Biswas

171 et al, 2021).

172 To produce phase-locked dynamics from a pair of oscillators with unequal natural frequencies 173 requires a special kind of complex coupling strategy labelled as 'power coupling' (Biswas et al., 2021).

174 A pair of oscillators coupled through power coupling is defined as:

$$
\dot{z_{2}}=\left(\mu-\beta_{1}\left|z_{2}\right|^{2}+i \omega_{2}\right) z_{2}+W_{21} z_{1}^{\frac{\omega_{2}}{\omega_{1}}}
$$

177 where, $W_{12}=A_{12} e^{i \frac{\theta_{12}}{\omega_{2}}}, W_{21}=A_{21} e^{i \frac{\theta_{21}}{\omega_{1}}}$ represent the complex power coupling coefficients on the

178 feedforward and feedback branches. Considering $\theta_{12}=-\theta_{21}$, and $A_{12}=A_{21}$, it has been shown that

179 the pair of oscillators can phase-lock at any of the solutions of the equation: $\frac{\dot{\phi_{1}}}{\omega_{1}}-\frac{\dot{\phi_{2}}}{\omega_{2}}=0$ depending on

180 the initial condition. The analytic solution of this problem gets increasingly complicated as the number

181 of oscillators in the network increases. Another limitation of the original power-coupling strategy is

182 that it does not ensure synchronization while the coupled oscillators are entrained to a new frequency 183 of oscillation.

184 Considering a simplified scenario of a pair of Hopf oscillators coupled through weak unilateral 185 power coupling, the one receiving input from the other oscillator through power coupling is being 186 perturbed by a strong complex sinusoidal external input signal with frequency close to the natural 187 frequency of the oscillator as depicted in fig. 2A. 


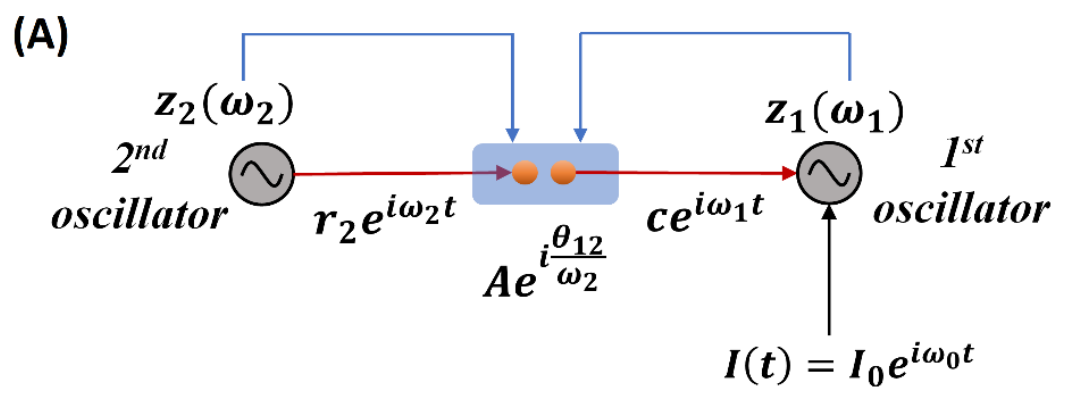

(B)

189 Figure 2: (A) The schematic diagram of a pair of Hopf oscillators, unilaterally coupled through

190 conventional power coupling strategy. Here $c$ is typically $A\left(\frac{\mu}{\beta_{1}}\right)^{\frac{\omega_{1}}{2 \omega_{2}}}$ at steady state. (B) The

191 fundamental building block of the OTSOM model. The single CAO oscillator receives inputs from two

192 sources: one from the SRO and the other from the external input signal. The two differences between

193 these two frameworks are the coupling coefficients; the natural frequency of the SRO is eliminated

194 from the denominator of the angle of the coupling coefficient, and the second being the actual

195 frequency of oscillation of the CAO oscillator is used to readjust the complex activation of the SRO

196 (subplot A) instead of the natural frequency of the post-synaptic oscillator (subplot B).

198 We will now try to show the pair of oscillators with an external sinusoidal input as shown in fig. 2A, 199 poses a new difficulty in phase-locking not faced by a pair of oscillators with power coupling (eqns. $4 a, b)$. Consider the situation in fig. $2 \mathrm{~A}$, where the $2^{\text {nd }}$ Oscillator sends a unilateral power coupling connection to the $1^{\text {st }}$ Oscillator, which in addition receives a complex sinusoidal signal of frequency $\omega_{0}$ as external input. Note that though the output of the $2^{\text {nd }}$ Oscillator has the frequency $\omega_{2}$, after the power coupling connection, the signal frequency changes to $\omega_{1}$. Thus the $1^{\text {st }}$ Oscillator receives two sinusoidal inputs - of frequencies $\omega_{0}$ and $\omega_{1}$. Therefore, the $1^{\text {st }}$ Oscillator does not simply entrain to the external input, due to the interference from the $2^{\text {nd }}$ Oscillator. In order to fix this problem, it turns out that we need a more general power coupling rule than the original one. 


\section{Modified Power Coupling}

To make the original power-coupling rule (Biswas et al 2021) more generalized, and to ensure synchronization in a pair of power-coupled oscillators even after entrainment, a modified version of the power-coupling strategy is proposed. For a pair of bilaterally coupled Hopf oscillators, the modified power-coupling mechanism is given as,

$$
\dot{z}_{1}=\left(\mu-\beta_{1}\left|z_{1}\right|^{2}+i \omega_{1}\right) z_{1}+W_{12} z_{2}{ }^{\frac{\omega_{1}^{*}}{\omega_{2}^{*}}}
$$

$$
\dot{z}_{2}=\left(\mu-\beta_{1}\left|z_{2}\right|^{2}+i \omega_{2}\right) z_{2}+W_{21} z_{1} \frac{\omega_{1}^{*}}{\omega_{2}^{*}}
$$

214 where, $W_{12}=A_{12} e^{i \frac{\theta_{12}}{\omega_{2}^{*}}}, W_{21}=A_{21} e^{i \frac{\theta_{21}}{\omega_{1}^{*}}}$, the only modification being $\omega_{1}^{*}$ and $\omega_{2}^{*}$ are actual frequencies

215 of oscillation instead of natural frequencies. A new set of dynamic equations (eqns. 8a,b) define $\omega_{1}^{*}$

216 and $\omega_{2}^{*}$. The overall dynamics of a pair of Hopf oscillators coupled through modified power-coupling 217 is represented in polar coordinates as follows.

$$
\dot{r}_{1}=\left(\mu-\beta_{1} r_{1}{ }^{2}\right) r_{1}+A_{12} r_{2}{ }^{\frac{\omega_{1}^{*}}{\omega_{2}^{*}}} \cos \omega_{1}^{*}\left(\frac{\theta_{12}}{\omega_{1}^{*} \omega_{2}^{*}}+\frac{\emptyset_{2}}{\omega_{2}^{*}}-\frac{\emptyset_{1}}{\omega_{1}^{*}}\right)
$$

$$
\dot{\emptyset}_{1}=\omega_{1}+A_{12} \frac{r_{2} \frac{\omega_{1}^{*}}{\omega_{2}^{*}}}{r_{1}} \sin \omega_{1}^{*}\left(\frac{\theta_{12}}{\omega_{1}^{*} \omega_{2}^{*}}+\frac{\emptyset_{2}}{\omega_{2}^{*}}-\frac{\emptyset_{1}}{\omega_{1}^{*}}\right)
$$

$$
\dot{r_{2}}=\left(\mu-\beta_{1} r_{2}{ }^{2}\right) r_{2}+A_{21} r_{1}{ }^{\frac{\omega_{2}^{*}}{\omega_{1}^{*}}} \cos \omega_{2}^{*}\left(\frac{\theta_{21}}{\omega_{1}^{*} \omega_{2}^{*}}+\frac{\emptyset_{1}}{\omega_{1}^{*}}-\frac{\emptyset_{2}}{\omega_{2}^{*}}\right)
$$

$$
\dot{\phi}_{2}=\omega_{2}+A_{21} \frac{r_{1}{ }^{\frac{\omega_{2}^{*}}{\omega_{1}^{*}}}}{r_{2}} \sin \omega_{2}^{*}\left(\frac{\theta_{21}}{\omega_{1}^{*} \omega_{2}^{*}}+\frac{\emptyset_{1}}{\omega_{1}^{*}}-\frac{\emptyset_{2}}{\omega_{2}^{*}}\right)
$$

$$
\tau_{\omega} \dot{\omega}_{1}^{*}=-\omega_{1}^{*}+\omega_{1}+A_{12} \frac{r_{2}^{\frac{\omega_{1}^{*}}{\omega_{2}^{*}}}}{r_{1}} \sin \omega_{1}^{*}\left(\frac{\theta_{12}}{\omega_{1}^{*} \omega_{2}^{*}}+\frac{\emptyset_{2}}{\omega_{2}^{*}}-\frac{\emptyset_{1}}{\omega_{1}^{*}}\right)
$$

$$
\tau_{\omega} \dot{\omega}_{2}^{*}=-\omega_{2}^{*}+\omega_{2}+A_{21} \frac{r_{1}^{\frac{\omega_{2}^{*}}{\omega_{1}^{*}}}}{r_{2}} \sin \omega_{2}^{*}\left(\frac{\theta_{21}}{\omega_{1}^{*} \omega_{2}^{*}}+\frac{\emptyset_{1}}{\omega_{1}^{*}}-\frac{\emptyset_{2}}{\omega_{2}^{*}}\right)
$$


224 where, $\tau_{\omega}$ is the time constant for $\omega_{1}^{*}$ and $\omega_{2}^{*}$. It is evident that without eqns. $8 \mathrm{a}$ and $8 \mathrm{~b}$, the modified 225 power-coupling is functionally the same as the conventional one as $\omega^{*}$ remains $\omega$. It can also be 226 observed that neither of the oscillators can get entrained to a new frequency of oscillation, which makes 227 a pair of bilaterally coupled Hopf oscillators through modified power coupling functionally the same 228 as a pair of bilaterally coupled Hopf oscillators through conventional power coupling (refer to 229 Appendix 1).

230 In the subsequent sections, the details of the intrinsic dynamics and the training framework of OSTOM model is described. The dynamics of a typical CAO oscillator is given as,

The first two terms on the RHS denote the intrinsic dynamics of the Hopf oscillator, the third term

234 represents the input from SRO $\left(I_{r}=W_{i j}^{r} z_{r} \frac{\omega_{i j}^{*}}{\omega_{r}}\right)$, the fourth term represents the aggregate external input $235\left(I_{e}=\varepsilon I(t)\right)$, where $I(t)$ is the actual external input. Note that only the SRO input is given via modified power coupling whereas the external input $I(t)$ is presented directly with a multiplicative factor, $\varepsilon$.

Similarly, the dynamics of the SRO is given as,

$$
\dot{z}_{r}=\left(\mu_{r}-\beta_{1 r}\left|z_{r}\right|^{2}\right) z_{r}+i \omega_{r} z_{r}
$$

239 The activation of the CAO oscillator at location $(i, j)$ is defined as, $z_{i j}=x_{i j}+i y_{i j}=r_{i j} e^{i \emptyset_{i j}}$; 240 similarly, the activation of the SRO oscillator is $z_{r}=x_{r}+i y_{r}=r_{r} e^{i \emptyset_{r}}$. Intrinsic dynamics of the CAO 241 oscillator is defined by the parameters $\mu, \beta_{1}$ and $\omega_{i j}$. Note that $\mu$ and $\beta_{1}$ are the same for all CAO 242 oscillators but $\omega_{i j}$ is different. The intrinsic dynamics of the SRO oscillator is defined by $\mu_{r}, \beta_{1 r}$ and $243 \omega_{r}$ parameters. $W_{i j}^{r}$ is the complex power-coupling weight from the reference oscillator to the oscillator 244 at $(i, j)$ in CAO, where $W_{i j}^{r}=A_{i j}^{r} e^{i \theta_{i j}^{r}}$.

245 The reason behind dropping the actual frequency of the presynaptic oscillator from the denominator of 246 the angle of the complex coupling coefficient will be justified in the following sections.

247 The Cartesian and the polar coordinate representations are respectively. 


$$
\begin{aligned}
& \dot{x_{l J}}=\left(\mu-\beta_{1}\left(x_{i j}^{2}+y_{i j}^{2}\right)\right) x_{i j}-\omega_{i j} y_{i j}+A_{i j}^{r}\left(x_{r}^{2}+y_{r}^{2}\right)^{\frac{\omega_{i j}^{*}}{2 \omega_{r}}} \cos \left(\theta_{i j}^{r}+\frac{\omega_{i j}^{*}}{\omega_{r}} \tan ^{-1} \frac{y_{r}^{2}}{x_{r}^{2}}\right)+\varepsilon \operatorname{imag}(I(t)) \\
& y_{\imath J}=\left(\mu-\beta_{1}\left(x_{i j}^{2}+y_{i j}^{2}\right)\right) y_{i j}+\omega_{i j} x_{i j}+A_{i j}^{r}\left(x_{r}^{2}+y_{r}^{2}\right)^{\frac{\omega_{i j}^{*}}{2 \omega_{r}}} \sin \left(\theta_{i j}^{r}+\frac{\omega_{i j}^{*}}{\omega_{r}} \tan ^{-1} \frac{y_{r}^{2}}{x_{r}^{2}}\right)+\varepsilon \operatorname{real}(I(t)) \\
& \dot{x_{r}}=\left(\mu_{r}-\beta_{1 r}\left(x_{r}^{2}+y_{r}^{2}\right)\right) x_{r}-\omega_{r} y_{r} \\
& \dot{y}_{r}=\left(\mu_{r}-\beta_{1 r}\left(x_{r}^{2}+y_{r}^{2}\right)\right) y_{r}+\omega_{r} x_{r} \\
& r_{\imath j}=\left(\mu-\beta_{1} r_{i j}^{2}\right) r_{i j}+A_{i j}^{r} r_{r}^{\frac{\omega_{i j}^{*}}{\omega_{r}}} \cos \omega_{i j}^{*}\left(\frac{\theta_{i j}^{r}}{\omega_{i j}^{*}}+\frac{\emptyset_{r}}{\omega_{r}}-\frac{\emptyset_{i j}}{\omega_{i j}^{*}}\right)+\operatorname{creal}\left(I(t) e^{-i \emptyset_{i j}}\right) \\
& \dot{\emptyset}_{l j}=\omega_{i j}+A_{i j}^{r} \frac{r_{r}^{\frac{\omega_{i j}^{*}}{\omega_{r}}}}{r_{i j}} \sin \omega_{i j}^{*}\left(\frac{\theta_{i j}^{r}}{\omega_{i j}^{*}}+\frac{\emptyset_{r}}{\omega_{r}}-\frac{\emptyset_{i j}}{\omega_{i j}^{*}}\right)+\frac{\varepsilon}{r_{i j}} \operatorname{imag}\left(I(t) e^{-i \emptyset_{i j}}\right) \\
& \dot{r}_{r}=\left(\mu_{r}-\beta_{1 r} r_{r}^{2}\right) r_{r} \\
& \dot{\phi}_{r}=\omega_{r} \\
& \tau_{\omega} \omega_{\imath j}^{*}=-\omega_{i j}^{*}+\omega_{i j}+A_{i j}^{r} \frac{r_{r}^{\frac{\omega_{i j}^{*}}{\omega_{r}}}}{r_{i j}} \sin \omega_{i j}^{*}\left(\frac{\theta_{i j}^{r}}{\omega_{i j}^{*}}+\frac{\emptyset_{r}}{\omega_{r}}-\frac{\emptyset_{i j}}{\omega_{i j}^{*}}\right)+\frac{\varepsilon}{r_{i j}} \operatorname{imag}\left(I(t) e^{-i \emptyset_{i j}}\right)
\end{aligned}
$$

The uniform, real-valued, afferent connections from the external input $(I(t))$ to the CAO oscillators is $\varepsilon . \omega_{i j}^{*}$ and $\omega_{r}^{*}$ are the actual frequencies of the CAO oscillators and the SRO respectively. As $\omega_{r}^{*}$ remains $\omega_{r}$, it is replaced with $\omega_{r}$. The actual frequency of oscillation of the CAO oscillator can be entrained to the frequency of the external perturbation. This entrainment property of the Hopf oscillator is utilized to realize the framework of the proposed model which will be discussed in detail in the later sections. Before elaborating the details of the training framework of the OTSOM model we are going the briefly analyze the intrinsic dynamical properties of the single unit (the SRO unilaterally coupled to a CAO oscillator) of the model.

\section{Dynamical response of a single unit:}

266 The single unit, which is the fundamental building block of the whole OSTOM model is constituted of 
and, 2) from the external input. The SRO is coupled to a CAO oscillator unilaterally through a modified power coupling connection (eqns. 9,10,11). The output of the SRO, after passing through modified power-coupling connection, takes on the same frequency as that of the CAO oscillator it projects to.

The CAO oscillator is also driven by external input signal through a real-valued afferent connection (fig. 2B). The external input is a complex sinusoidal signal.

We now investigate the steady state response of the single unit by analyzing (eqns. 12,13,14). Let the external input signal, $I(t)=I_{0} e^{i \emptyset_{0}}=I_{0} e^{i\left(\omega_{0} t+\xi_{0}\right)}$ where $\omega_{0}$ and $\xi_{0}$ are respectively the frequency and the phase offset of complex sinusoidal signal. Introducing the following variables,

- $\quad$ the relative phase of the $\mathrm{CAO}$ oscillator w.r.t the input, $\psi=\varnothing-\emptyset_{0}$,

- relative frequency of the CAO oscillator w.r.t the input, $\Omega=\omega-\omega_{0}$, and

- the normalized phase difference between the CAO oscillator and the SRO, $\lambda_{r}=\frac{\emptyset}{\omega^{*}}-\frac{\emptyset_{r}}{\omega_{r}}$,

$$
\dot{r}=\left(\mu-\beta_{1} r^{2}\right) r+A_{r} r_{r}^{\frac{\omega^{*}}{\omega_{r}}} \cos \left(\theta_{r}-\lambda_{r} \omega^{*}\right)+\varepsilon I_{0} \cos \psi
$$

$$
\dot{\psi}=\Omega_{i j}+A_{r} \frac{r_{r}^{\frac{\omega^{*}}{\omega_{r}}}}{r} \sin \left(\theta_{r}-\lambda_{r} \omega^{*}\right)-\frac{\varepsilon I_{0}}{r} \sin \psi
$$

$$
\dot{r}_{r}=\left(\mu_{r}-\beta_{1 r} r_{r}^{2}\right) r_{r}
$$

$$
\dot{\emptyset}_{r}=\omega_{r}
$$

Under the special condition, $0<\varepsilon \ll 1$, the single unit is equivalent to a pair of unidirectionally coupled oscillators through modified power coupling. There are two differences between the modified power coupling used under unilateral coupling scenario in the present study and the conventional power coupling proposed in (Biswas et al., 2021). In the conventional power coupling scheme of (Biswas et al 2021), the complex state of the oscillator is raised to the ratio of the intrinsic frequencies of the presynaptic and postsynaptic oscillators. In the modified power coupling proposed now, the exponent 
292 frequency of oscillation of the presynaptic oscillator (denoted by dynamical variable $\omega^{*}$ as defined by 293 eqns. 11 and 14). The second difference is that, in modified power coupling, the angle of the complex 294 power coupling coefficient does not incorporate the natural frequency of the presynaptic oscillator in 295 the denominator (conventional and modified power coupling coefficients are: $W_{i j}=A_{i j} e^{i \frac{\theta_{i j}}{\omega_{j}}}$ and $296 W_{i j}=A_{i j} e^{i \theta_{i j}}$ respectively). At steady state the normalized phase difference between the two 297 oscillators $\left(\frac{\emptyset}{\omega^{*}}-\frac{\emptyset_{r}}{\omega_{r}}\right)$ will be $\frac{1}{\omega}$ times the angle of the complex coupling coefficient $\left(\theta_{r}\right)$ (see Appendix2981 for proof).

299 Under normal condition $(\varepsilon \neq 0)$ we are going to consider the special scenario where $\omega_{0}$ falls under the 300 entrainment regime of the $\mathrm{CAO}$ oscillator. The entrainment regime of an individual oscillator, 301 receiving only $\varepsilon I(t)$ as input, denotes the range of values of $\Omega$ for which the Hopf oscillator exhibits 302 either stable fixed-point or stable spiral behavior in $(r, \psi)$ space under the influence of $I(t)$. Inside 303 entrainment regime the actual frequency of oscillation $\left(\omega^{*}\right)$ of the oscillator is entrained to the 304 frequency of the input signal $\left(\omega_{0}\right)$ if the natural frequency of the Hopf oscillator $(\omega)$ is sufficiently 305 close to $\omega_{0}$. (i.e., $|\Omega|$ is sufficiently small). In fig. 3 the entrainment regime can be identified as the 306 purple region as a function of $\mu, \beta_{1}$ and the intensity of the driving input $\left(\varepsilon I_{0}\right)$. The boundary of the 307 entrainment regime on these parameter space can also be identified as an analytical expression. From 308 Appendix-2 we found out that the steady-state phase offset of the oscillator inside the entrainment 309 regime is: $\xi_{0}+\sin ^{-1} \frac{\Omega r_{S S}}{\varepsilon I_{0}}$. At the boundary of the entrainment regime the argument of the $\arcsin$ 310 operator is 1. i.e., $\frac{\Omega r_{S S}}{\varepsilon I_{0}}=1$, which is presented in figs. $3 \mathrm{D}, \mathrm{E}, \mathrm{F}$ as a function of one of these three 311 parameters keeping other two fixed. 

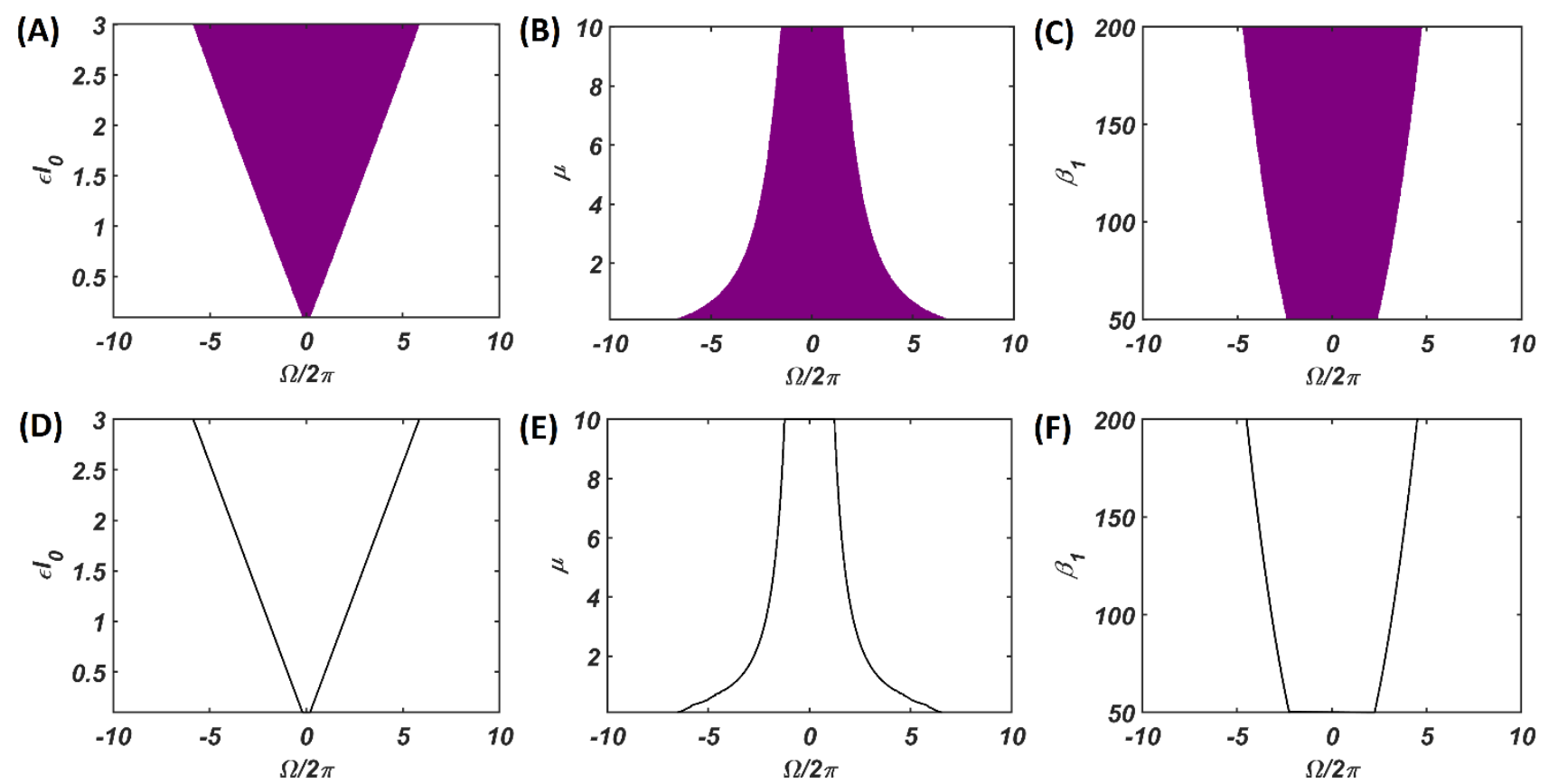

313 Figure 3: The purple region on the (A), (B) and (C) plots shows the entrainment regime of an

314 individual Hopf oscillator and how it is dependent on its intrinsic dynamical parameters $\left(\mu\right.$ and $\left.\beta_{1}\right)$ and

315 the intensity of the driving input $\left(\varepsilon I_{0}\right)$. Whereas the boundaries of the entrainment regime on these

316 parameter space plotted in (D), (E) and (F), represented by the function $\frac{\Omega r_{S S}}{\varepsilon I_{0}}=1$. In each of these plots

317 one of these parameters are varied while keeping others fixed at: $\mu=1, \beta_{1}=150, \varepsilon I_{0}=2$.

318 The dependency of the width of the entrainment regime, $\Delta \omega$ on $\mu, \beta_{1}$ and $\varepsilon I_{0}$ is further illustrated in

319 fig. A2.2 in the Appendix. Although at the beginning, the CAO oscillator is perturbed by two complex

320 sinusoidal input signals, one with frequency $\omega$ (from the SRO, but after the modified power-coupling

321 step) and the other with frequency $\omega_{0}$ (external input $I(t)$ ), it is entrained to the external input signal

322 because of the dominance of the perturbation by $I(t)$ over the perturbation caused by the input from

323 SRO since we assume that $A_{r}<\varepsilon I_{0}$. The entrainment width of the CAO oscillator will also depend on

$324 \theta_{r}-\xi_{0}$ (fig. 6). Since the SRO does not receive any inputs, it always oscillates at its natural frequency

325 of oscillation $\left(\omega_{r}\right)$.

Thus, under the conditions of entrainment, a given CAO oscillator gets two complex sinusoidal signals as inputs with the same frequency as $I(t)$, with different magnitude and phase offsets, given as follows: 
- 1) the $3^{\text {rd }}$ term in the RHS of eqn. 9 evolves to $W_{i j}^{r} z_{r}^{\frac{\omega_{i j}^{*}}{\omega_{r}}}=A_{i j}^{r} r_{r} \frac{\omega_{i j}^{*}}{\omega_{r}} e^{i\left(\theta_{i j}^{r}+\emptyset_{r} \frac{\omega_{i j}^{*}}{\omega_{r}}\right)}=$ $A_{i j}^{r} r_{r}^{\frac{\omega_{0}}{\omega_{r}}} e^{i\left(\theta_{i j}^{r}+\emptyset_{r} \frac{\omega_{0}}{\omega_{r}}\right)}=A_{i j}^{r} r_{r}^{\frac{\omega_{0}}{\omega_{r}}} e^{i\left(\omega_{0} t+\theta_{i j}^{r}\right)}$ at steady state (refer to Appendix-4), denoted by $I_{r}(t)=a_{r} e^{i\left(\omega_{0} t+\xi_{r}\right)}$ and,

- 2) the $4^{\text {th }}$ term in RHS of eqn. $9, \varepsilon I_{0} e^{i\left(\omega_{0} t+\xi_{0}\right)}$ denoted by $I_{e}(t)=a_{0} e^{i\left(\omega_{0} t+\xi_{0}\right)}$ (Appendix-4), where $a_{r}$ is dependent on the steady state magnitude of oscillation of the reference oscillator $\left(r_{r s s}\right)$, which in turn depends on $\mu_{r}, \beta_{1 r}$ and $A_{r} . \xi_{r}$ being $\theta_{r}$ justifies why the actual frequency of the presynaptic oscillator is omitted from denominator of the angle of the modified power coupling coefficient. Both of these inputs either constructively or destructively interfere with each other depending on their relative phase offset $\left(\xi_{r}-\xi_{0}=\theta_{r}-\xi_{0}\right)$. Since the magnitude of the response of the $\mathrm{CAO}$ oscillator is either diminished or increased, depending on the relative values of $\theta_{r}$ and $\xi_{0}$, with this arrangement the CAO oscillator will be capable of encoding phase offset of the complex sinusoidal input signal.

(A)

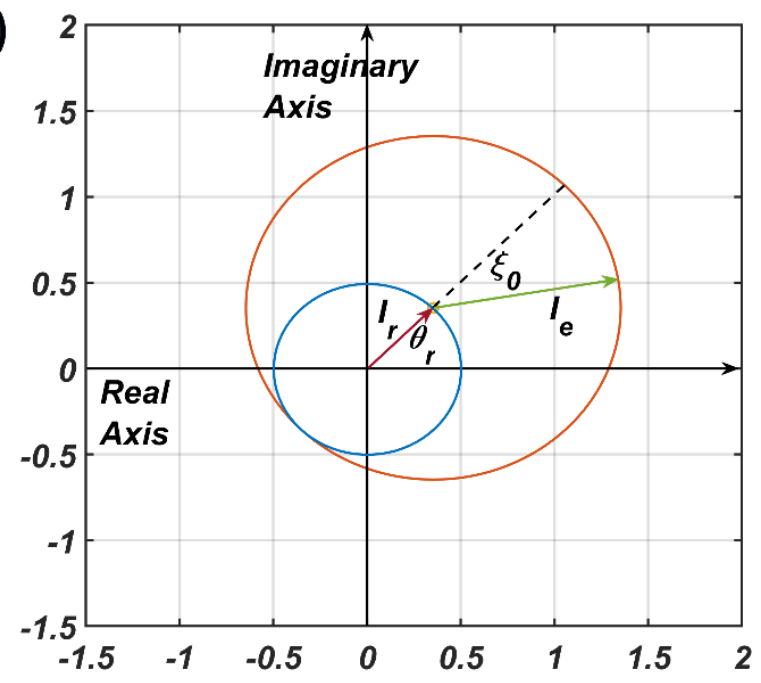

(B)

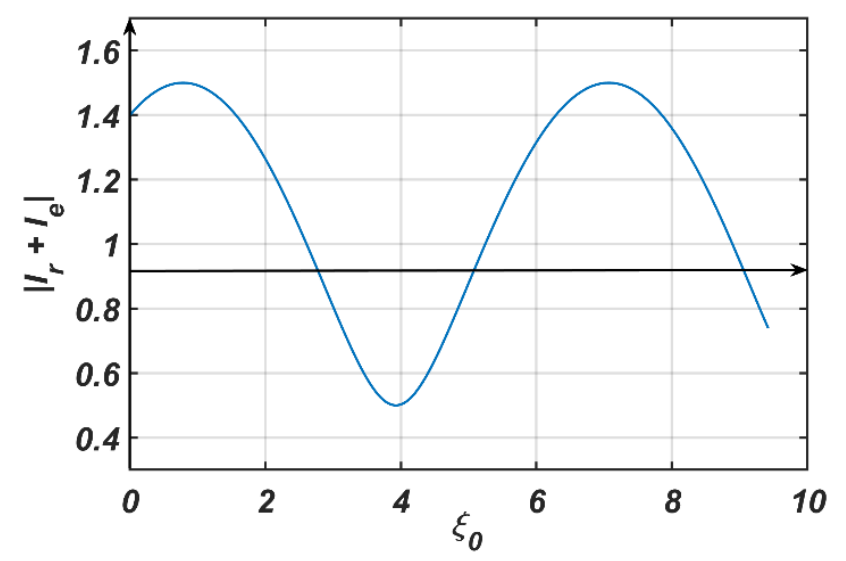

342 Figure 4: The pictorial representation of the constructive and destructive interference between the two

343 inputs to a CAO oscillator. $I_{r}=0.5 e^{i \theta_{r}}, I_{e}=e^{i \xi_{0}}$ are the phasors of the input from the reference 344 oscillator and the external input respectively. $I=I_{e}+I_{r}$. In (B) $\theta_{r}$ is kept fixed at $\frac{\pi}{4}$ and $\xi_{0}$ is varied 345 to plot $|I|$ from which it can be observed that $|I|$ is maximum when $\xi_{0}=2 n \pi+\theta_{r}$ and minimum when $346 \xi_{0}=(2 n+1) \pi+\theta_{r}$.

\section{Modified Hebbian learning:}


A modified Hebbian learning rule is proposed for training the modified power coupling described in the previous section. The complex variable and the polar coordinate version of the modified Hebbian learning rule is described as following;

$$
\tau_{W} \dot{W}_{r}=-W_{r}+z\left(\bar{z}_{r}\right)^{\frac{\omega^{*}}{\omega_{r}}}
$$

$$
\tau_{W} \dot{A_{r}}=-A_{r}+r r_{r} \frac{\omega^{*}}{\omega_{r}} \cos \omega^{*}\left(\frac{\emptyset}{\omega^{*}}-\frac{\theta_{r}}{\omega^{*}}-\frac{\emptyset_{r}}{\omega_{r}}\right)
$$

$$
\boldsymbol{\tau}_{W} \dot{\boldsymbol{\theta}_{r}}=\frac{\boldsymbol{r} \boldsymbol{r}_{r}^{\frac{\omega^{*}}{\omega_{r}}}}{\boldsymbol{A}_{r}} \sin \omega^{*}\left(\frac{\emptyset}{\omega^{*}}-\frac{\boldsymbol{\theta}_{r}}{\omega^{*}}-\frac{\emptyset_{r}}{\omega_{r}}\right)
$$

The modified Hebbian learning rule as prescribed in eqns. 15a,b can be compared to the original Hebbian learning for the power coupling coefficient proposed earlier (eqn. 15 in Biswas et al., 2021). Here $\overline{z_{r}}$ is the complex conjugate of the complex activation of the SRO. It can be observed that the modified Hebbian learning rule for the modified power coupling coefficient has a similar effect as the original Hebbian rule had in case of the previously proposed power coupling strategy.

Effectively the modified Hebbian learning rule is the same as the previous one when there is no entrainment. Without entrainment when the phase offset of the post-synaptic oscillator is driven to the phase offset of the complex sinusoidal input signal with identical frequency as the natural frequency of the CAO oscillator, $\theta_{r}$ learns $\xi_{0}$, where $\xi_{0}$ is the phase offset of the input driving the post-synaptic oscillator (refer to Appendix 5). Similarly, it can be shown that $A_{r}$ learns $r_{s s} r_{r s s} \frac{\omega}{\omega_{r}}$ (refer to Appendix 6 ), where $r_{s s}$ and $r_{r s s}$ are the steady state values of amplitude of oscillation of the post-synaptic and presynaptic oscillators respectively. With entrainment of the main oscillator $\theta_{r}$ learns $\xi_{0}+\sin ^{-1} \frac{\Omega r_{S S}}{\varepsilon I_{0}}$, which is the phase offset of the main oscillator at steady-state after entrainment while the Hebbian dynamics is enabled.

\section{Training the OTSOM:}

In the previous section, we discussed the response properties of a single CAO oscillator to its two inputs. We now discuss the training methodology of OTSOM. The OTSOM model is trained on complex sinusoidal signals, each defined by a characteristic frequency $\left(\omega_{0}\right)$ and phase $\left(\xi_{0}\right)$ (defined w.r.t. SRO). Thus, for a given sinusoidal input, we expect the OTSOM to produce a single winner, 
373 such that the row number, $i$, of the winner represents the input phase, while the column number, $j$,

374 represents the input frequency. Therefore, the CAO oscillator at $(i, j)$ gives maximum resonating 375 response when $\omega_{0}=\omega_{i j}$ and $\xi_{0}=\theta_{i j}^{r}$ which is why we have chosen to train the $\omega_{i j}$ and $\theta_{i j}^{r}$ parameters 376 of the OTSOM network. These parameters are trained in two consecutive training stages. In the $1^{\text {st }}$ 377 stage, $\omega_{i j}$ s are trained keeping $\theta_{i j}^{r}$ 's fixed and in the $2^{\text {nd }}$ stage, $\theta_{i j}^{r}$ s are trained by keeping the already 378 trained $\omega_{i j}$ s fixed.

\section{First Stage of training: Training Frequency}

380 In this stage, the natural frequencies, $\omega_{i j}$, of the CAO oscillators are trained according to a learning 381 rule analogous to the self-organizing map learning rule (Kohonen, 1990). Specifically, to train the 382 frequencies of the individual oscillators, we use the adaptive frequency Hopf oscillator model proposed 383 earlier (Biswas et al., 2021; Righetti et al., 2005). The training takes place over multiple epochs $384\left(N_{\text {epoch, } \omega}\right)$. In every epoch, $N$ input signals are randomly chosen from the input set $U$. The input set 385 contains complex sinusoidal signals with frequencies and phase offset sampled from a uniform 386 probability distribution. Once an input signal with certain frequency and phase offset $\left(I_{0} e^{i\left(\omega_{0 p} t+\xi_{0 p}\right)}\right)$

387 is selected, it is presented as the external input signal $\left(I_{p}(t)\right)$ to the oscillators in the CAO. After an 388 initial transient phase, which lasts for $T_{s \omega}$ seconds, the CAO oscillator response reaches steady state. 389 The oscillator with the largest amplitude at the end of transient phase is denoted the 'winner CAO 390 oscillator'. 
(A) Training Algorithm of OTSOM model:

- Construct a dynamical network defined by eqn. 9 and 10

- Initialize $\omega_{i j}$ 's randomly between $\omega_{\max }$ to $\omega_{\min }$

- Initialize $\theta_{i j}^{r}$ 's randomly between 0 to $2 \pi$

First stage of training: Training frequency

- Set $A_{i j}^{r} \ll \varepsilon$

For iepoch $=1, N_{\text {epoch, } \omega}$ do

For pattern $=1, N$ do and $U_{\xi}$

- Sample $\omega_{0 p}$ and $\xi_{0 p}$ from the Input sets $U_{\omega}$

Transient phase

- Simulate the network dynamics for $T_{s \omega}$ secs

- Choose the winner oscillator $z_{m n}$, which satisfies $\max _{i j}\left(r_{i j, s s}\right)$

Adaptive Hopf phase

- Define neighbourhood function w.r.t the winner $W_{i j}^{m n}$ and corresponding $\eta_{\omega_{i j}^{m n}}^{m n}$

- Train $\omega_{i j}$ 's using eqn. 16 for $T_{t \omega}$ secs

\section{End For}

Update $\sigma_{x \omega}, \sigma_{y \omega}$ (Annealing)

\section{End For}

(B) Second stage of training: Training phase

Adapt $\omega_{i j}$ 's learnt from the first episode

For iepoch $=1, N_{\text {epoch }, \theta}$ do

For pattern $=1, N$ do

- Sample $\omega_{0 p}$ and $\xi_{0 p}$ from the Input sets $U_{\omega}$ and $U_{\xi}$

Transient phase

- Set $\epsilon_{\max }>\varepsilon I_{0}-A_{i j}^{r}>\epsilon_{\text {min }}$

- Simulate the network dynamics for $T_{S \theta}$ secs

- Choose the winner oscillator $z_{m n}$, which satisfies $\max _{i j}\left(r_{i j, s s}\right)$

\section{Hebbian Plasticity phase}

- $\operatorname{Set} A_{i j}^{r} \ll \varepsilon$

- Define neighbourhood function w.r.t the

winner $W_{i j}^{m n}$ and corresponding $\eta_{\theta i j}^{m n}$

- Train $\theta_{i j}^{r}$ 's using eqn. 17 for $T_{h \theta}$ secs

\section{End For}

Update $\sigma_{x \theta}, \sigma_{y \theta}$ (Annealing)

End For

Figure 5: Algorithms for the first (A) and the second (B) stage of training of OTSOM model.

The dynamics of eqns. $(9,10,11)$ is simulated with the special condition $A_{i j}^{r} \cong 0$ during the transient phase. Under this condition the steady state solution of CAO oscillator $\left(r_{i j s s}^{*}, \psi_{i j s s}^{*}\right)$ can either be a stable node, stable spiral or unstable spiral (Kim \& Large, 2015). Typically, inside the entrainment regime we get stable node or stable spiral as the solution. The parameters $\mu, \beta_{1}$ and $\varepsilon$ play a crucial role during this phase as they determine not only the entrainment width of the Hopf oscillator $(\Delta \omega)$ but also the duration of the transient period $\left(T_{s \omega}\right)$. As the oscillator with the highest steady state amplitude of oscillation $\left(\max \left(r_{i j s s}\right)\right)$ is chosen to be the winner, it is evident that the winner oscillator should satisfy the condition $\min _{i j}\left|\omega_{i j}-\omega_{0 p}\right|$. After the transient period, the frequencies $\omega_{i j} \mathrm{~s}$ of all the CAO oscillators within the neighbourhood window, along with the winner oscillator, are trained for $T_{t \omega}$ secs according to the following dynamics (Biswas et al., 2021):

$$
\dot{\omega}_{\imath j}=-\eta_{\omega_{i j}}^{m n}(t) \varepsilon I_{0} \sin \psi_{i j}
$$


405

406

407

408

412

where $\eta_{\omega_{i j}}^{m n}$ is the neighbourhood function, centered on the winner oscillator located on the $m^{\text {th }}$ row and $n^{\text {th }}$ column, defined as following:

$\eta_{\omega_{i j} m}^{m n}(t)=W_{i j}^{m n} \eta_{\omega_{0}} e^{-\frac{(i-m)^{2}}{\sigma_{y \omega}(t)}-\frac{(j-n)^{2}}{\sigma_{x \omega}(t)}}$
$\sigma_{y \omega}(t)=\sigma_{y \omega m} e^{-\frac{i_{i t e r}^{2}}{\sigma_{\sigma y \omega}}}$
$\sigma_{x \omega}(t)=\sigma_{x \omega m} e^{-\frac{i_{i t e r}^{2}}{\sigma_{\sigma x \omega}}}$
$W_{i j}^{m n}=1 \quad|i-m| \leq d_{r \omega}$ and $|j-n| \leq d_{c \omega}$
$=0 \quad$ otherwise

$W_{i j}^{m n}$ is termed as neighbourhood window. Here, $d_{r \omega}$ and $d_{c \omega}$ are the half-lengths of the neighbourhood window along rows and columns respectively. Effectively the purpose of the neighbourhood window is to constrain the learning confined to the neighbourhood of the winner oscillator. Additionally, it can be observed that the neighbourhood function, $\eta_{\omega_{i j}}^{m n}$, is a Gaussian centred on the winner oscillator. The standard deviations along the rows and columns $\left(\sigma_{y \omega}\right.$ and $\left.\sigma_{x \omega}\right)$ of $\eta_{\omega_{i j}}^{m n}$ decrease with time to produce annealing effect. Thus, the network dynamics is simulated for $\left(T_{s \omega}+T_{t \omega}\right)$ seconds for each presentation of a training signal, for $N\left(T_{s \omega}+T_{t \omega}\right)$ during each training epoch, where $\mathrm{N}$ is the number of training signals. Therefore, the time required for the entire first phase of training is $N_{\text {epoch, } \omega} N\left(T_{s \omega}+T_{t \omega}\right)$.

\section{Second stage training: training phase off-set}

While the objective of the first stage training is to train the frequencies, $\omega_{i j} \mathrm{~s}$, of the CAO oscillators, the objective of the second stage training is to train the phases of the same oscillators which are determined by the angles, $\theta_{i j}^{r}$, of feedforward connections from the SRO to the CAO oscillators. After the first stage training, $\omega_{i j}$ s self-organize in an increasing order along the rows, a pattern confirmed by the simulations shown in the results section. This occurs because the CAO is a rectangular array with the number of columns much larger than the number of rows. The $2^{\text {nd }}$ stage of training commences with randomly initialized $\theta_{i j}^{r}$ parameters and follows the algorithmic course as given in fig. 5B. 
In the second stage training, during the transient period, the magnitudes of $A_{i j}^{r}$ parameters are increased to the same order of magnitude as $\varepsilon$. The training takes place through multiple epochs $\left(N_{\text {epoch }, \theta}\right)$ in which, as in the previous stage, each training pattern is a complex sinusoidal signal with specific $\omega_{0 p}$ and $\xi_{0 p}$, sampled from the input set $U$. As in the previous training stage, the network dynamics is first allowed to reach the steady state before $\theta_{i j}^{r} \mathrm{~s}$ are adapted. The transient phase dynamics, given by eqns. $9,10,11$ is simulated for $T_{s \theta}$ secs. At the end of the transient period the winner oscillator is identified by its steady state amplitude of oscillation $\left(\max _{i j} r_{i j s s}\right)$. It is expected that the natural frequency $\left(\omega_{m n}\right)$ and the angle of the power coupling coefficient $\left(\theta_{m n}^{r}\right)$ of the winner oscillator should be closest to $\omega_{0 p}$ and $\xi_{0 p}$ respectively. During the subsequent $T_{h \theta}$ period $\theta_{i j}^{r}$ parameters of the unidirectional power coupling coefficients are trained according to the learning rule given in eqn-17. As eqn-17 is derived from the modified Hebbian learning rule as proposed in eqn$15 \mathrm{~b}$, this phase is termed as Hebbian learning phase.

$$
\begin{aligned}
& \dot{\theta}_{i j}^{r}=\eta_{\theta_{i j}}^{m n}(t) \frac{r_{i j} r_{r}^{\frac{\omega_{i j}^{*}}{\omega_{r}}}}{A_{i j}^{r}} \sin \omega_{i j}^{*}\left(\frac{\emptyset_{i j}}{\omega_{i j}^{*}}-\frac{\theta_{i j}^{r}}{\omega_{i j}^{*}}-\frac{\emptyset_{r}}{\omega_{r}}\right) \\
& \eta_{\theta_{i j}^{m n}}^{m n}(t)=W_{i j}^{m n}\left(\left(\eta_{\theta_{\max }}-\eta_{\theta_{\min }}\right) e^{-\frac{(i-m)^{2}}{\sigma_{y \theta}(t)}-\frac{(j-n)^{2}}{\sigma_{x \theta}(t)}}+\eta_{\theta_{\min }}\right)
\end{aligned}
$$

$$
\begin{gathered}
\sigma_{y \theta}(t)=\sigma_{y \theta m} e^{-\frac{i_{i t e r}^{2}}{\sigma_{\sigma y \theta}}} \\
\sigma_{x \theta}(t)=\sigma_{x \theta m} e^{-\frac{i_{i t e r}^{2}}{\sigma_{\sigma x \theta}}}
\end{gathered}
$$

Here, $m$ and $n$ denote the index of the winner oscillator. The gaussian shaped neighbourhood function, $\eta_{W i j}^{m n}$, is confined between $\eta_{W \max }$ and $\eta_{W \min }$. The learning neighbourhood window $W_{i j}^{m n}$ is defined w.r.t the winner oscillator similar to the first stage training. In the Hebbian learning phase $A_{i j}^{r}$ 448 parameters are not trained $\left(\dot{A_{l j}^{r}}=0\right)$, we have kept $A_{i j}^{r}$ values close to zero or $A_{i j}^{r} \ll \varepsilon I_{0}$. In which case, $\theta_{i j}^{r}$ learns $\xi_{0 p}+\sin ^{-1} \frac{\Omega_{i j}^{p} r_{i j, s s}}{\varepsilon I_{0}}$ at steady-state when the CAO oscillator receives the input $I_{0} e^{i\left(\omega_{0 p} t+\xi_{0 p}\right)}$ 450 (refer to Appendix-5) (where $\Omega_{i j}^{p}=\omega_{i j}-\omega_{0 p}$ ). It has also been shown that even if $A_{i j}^{r}$ is not negligible 
451 (i.e., $\left.\dot{A}_{l j}^{r}=0, A_{i j}^{r} \neq 0\right) \theta_{i j}^{r}$ learns $\xi_{0 p}+\sin ^{-1} \frac{\Omega_{i j}^{p} r_{i j, s s}}{\varepsilon I_{0}}$ at steady-state (refer to Appendix-6). This

452 happens because the phase offset of the CAO oscillator $\left(\delta_{i j}\right)$ attains the value $\xi_{0 p}+\sin ^{-1} \frac{\Omega_{i j}^{p} r_{i j, s s}}{\varepsilon I_{0}}$ at

453 steady state inside the entrainment regime. Each training epoch in the $2^{\text {nd }}$ stage training takes $454 N\left(T_{s \theta}+T_{h \theta}\right)$ seconds, and the time required for the entire $2^{\text {nd }}$ stage of training is $N_{\text {epoch, } \theta} N\left(T_{s \theta}+\right.$ $\left.455 T_{h \theta}\right)$.

\section{Results}

\section{Single oscillator results:}

459 We now numerically analyse the response of a single CAO oscillator to the simultaneously received 460 inputs from $\operatorname{SRO}\left(I_{r}(t)\right)$ and the external input $\left(I_{e}(t)\right)$ for the following conditions:

461 (a) $A_{r}$ being sufficiently smaller but not negligible w.r.t $\varepsilon I_{0}$ (i.e., the strength of the $I_{e}(t)$ combining 462 its own magnitude of oscillation and the afferent weight) which enables the CAO oscillator to get 463 entrained to $I(t)$, and ensures significant interference between the two inputs. The condition can be 464 mathematically rephrased as:

$$
\epsilon_{\max }>\varepsilon I_{0}-A_{r}>\epsilon_{\min }
$$

where $\epsilon_{\min }$ and $\epsilon_{\max }$ are positive numbers.

(b) $A_{r}$ is negligible w.r.t $\varepsilon I_{0}$, which is similar to the scenario where CAO oscillator is only perturbed by $I(t)$.

$$
A_{r} \ll \varepsilon I_{0}
$$

We have numerically analyzed the response of the CAO oscillator under the $1^{\text {st }}$ condition (eqn. 18a) as described by the eqns.- 9,10,11. The CAO oscillator exhibits stable entrainment depending on both the relative frequency $(\Omega)$ and the relative phase $\left(\theta_{r}-\xi_{0}\right)$ w.r.t the external input signal. When it shows stable entrainment, the magnitude of oscillation reaches a fixed value at steady state. There is a 474 distinguishable boundary on the $\Omega$ vs $\theta_{r}-\xi_{0}$ plane in which stable entrainment is observed. Outside 475 this boundary the CAO either exhibits intermittent entrainment or does not get entrained at all. In the 476 following figure (fig. 6) the region in the $\Omega$ vs $\theta_{r}-\xi_{0}$ plane where stable entrainment is observed is 
portrayed as purple region. It can be observed that there is a symmetry in the purple region w.r.t the

$478 \Omega=0$ and $\theta_{r}-\xi_{0}=0$ axis $\left(\theta_{r}=\pi\right)$. A pair of CAO oscillator and a SRO is simulated until the steady

479 state is achieved to find out the mean, maximum and the minimum value of the steady state magnitude

480 of oscillation of the CAO oscillator. The following parameters are used: $\mu=1, \beta_{1}=-100, \omega=$ $4812 \pi 60, \omega_{0}=2 \pi 50$ to $2 \pi 70, \xi_{0}=0$ to $2 \pi, \varepsilon=2, F=1, \mu_{r}=1, \beta_{1 r}=-10, \omega_{r}=2 \pi \times 60, \tau_{\omega}=$ $4820.1, A_{r}=0.5, \theta_{r}=\pi$ for the simulation results provided in the figures (figs. 7,8,9). The steady state 483 is attained typically before 3 secs. The steady state behaviour of the magnitude of oscillation of the 484 CAO oscillator for eight different symmetrical cross sections parallel to $\Omega=0$ axis and for another 485 eight different symmetrical cross sections parallel to $\theta_{r}-\xi_{0}=0$ axis are plotted in the fig. 7 and fig. 4868.

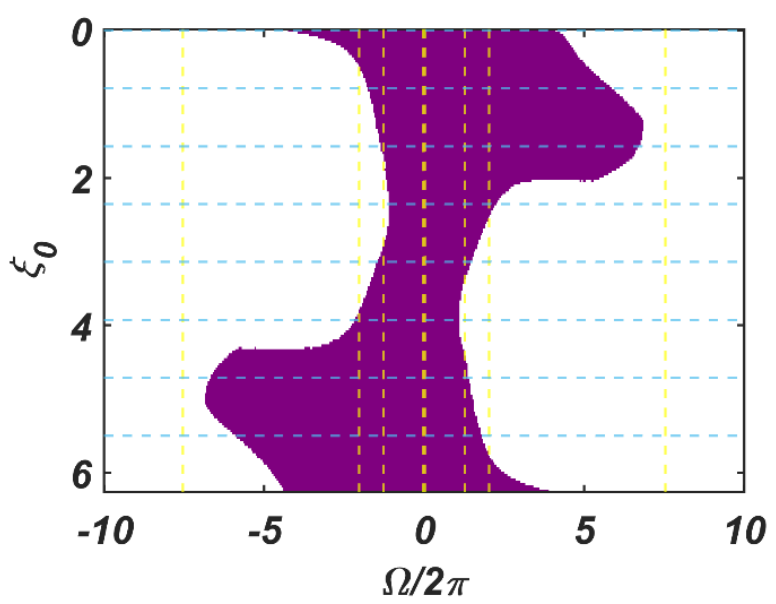

488 Figure 6: The figure principally depicts the entrainment regime (purple region) of the CAO oscillator 489 490 on the $\Omega$ vs $\theta_{r}-\xi_{0}$ plane, keeping the other factors such as $\varepsilon I_{0}, \theta_{r}, A_{r}$ and the intrinsic parameters of the CAO oscillator and the SRO fixed. 
(A)

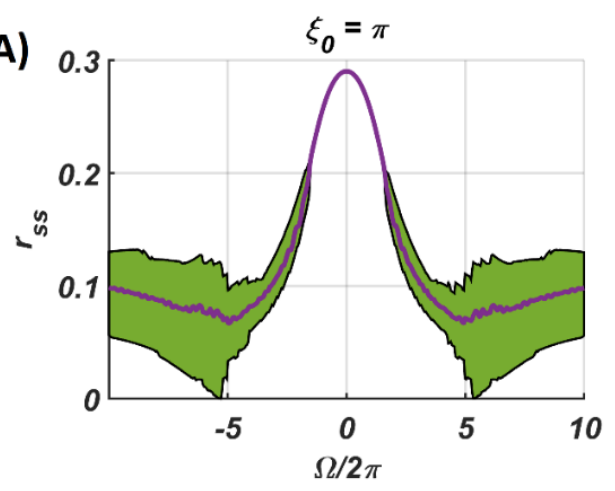

(C)

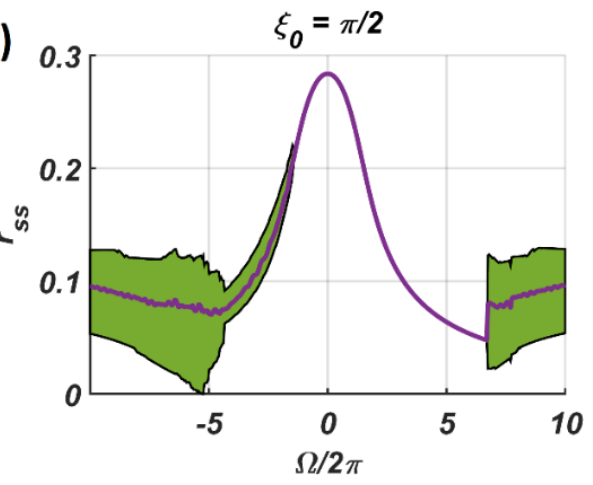

(E)

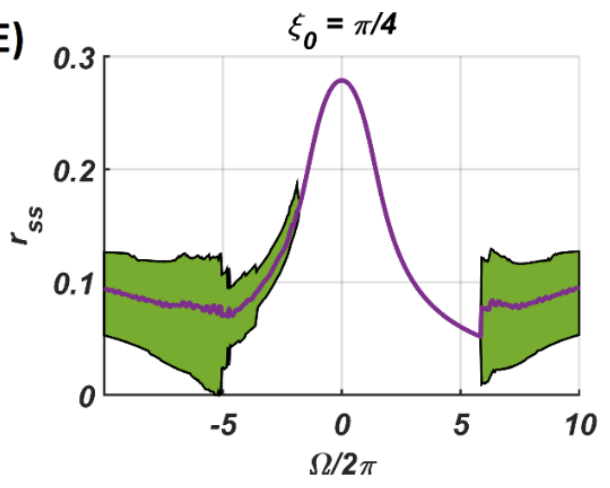

(G)

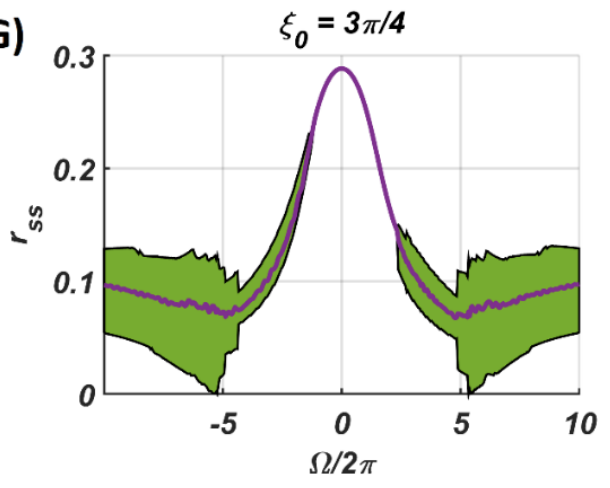

(B)

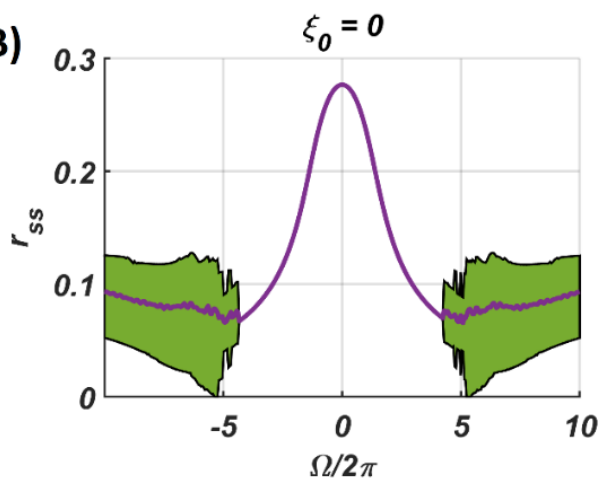

(D)

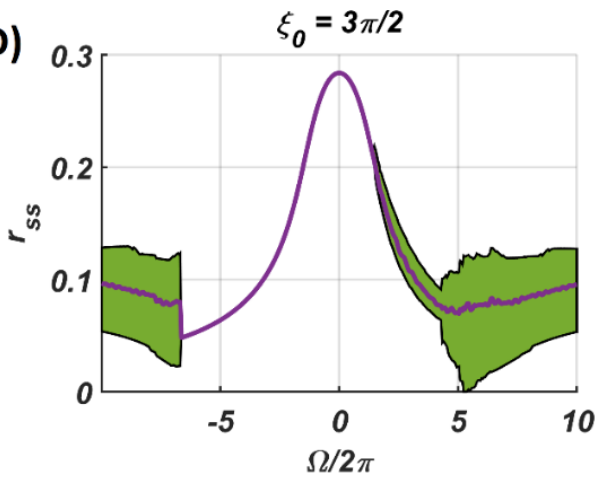

(F)

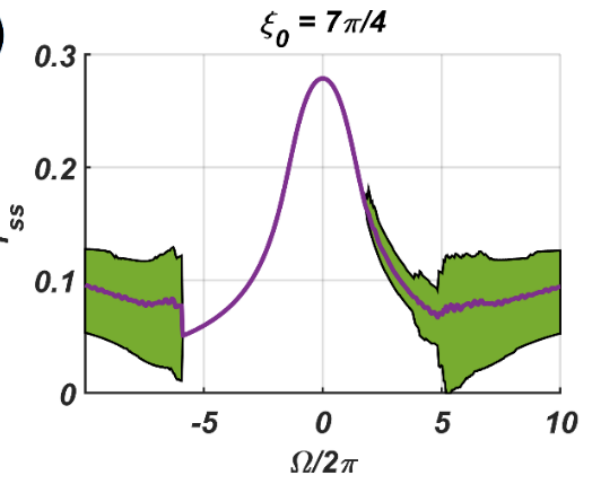

(H)

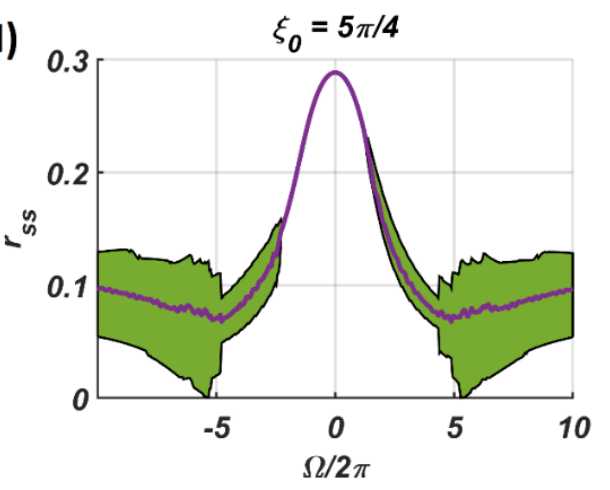

492 Figure 7: The steady-state magnitude of oscillation of the CAO oscillator with the identical pair of 493 coupled CAO oscillator and SRO as provided in the fig. 6 but for a symmetrical distinct value of the 494 phase offset of the external input signal w.r.t the angle of the power coupling connection $\theta_{r}=\pi$, cross 
495 section of which is drawn in blue lines in fig. 6. The green region depicts the variance of steady state 496 magnitude of oscillation w.r.t the mean value, plotted in purple.

497 In the figs. 8 and 9 the $r_{s s}$ is plotted on a same scale of magnitude so that the resonance exhibited by 498 the CAO oscillator w.r.t the frequency and the phase offset of the external input can be compared. It is 499 apparent that $\omega$ has a greater effect on CAO oscillator in terms of its steady-state magnitude of 500 oscillation than $\xi_{0}$. The reason behind introducing the lower bound on $\varepsilon I_{0}-A_{r}\left(\epsilon_{\min }\right)$ is justified here.

501 In other words, the resonance exhibited by the CAO oscillator w.r.t the $\xi_{0}$ at a smaller scale is ensured 502 by the condition $\varepsilon I_{0}-A_{r}>\epsilon_{\min }$. The combined results of figs. 7, 8, 9 reveals that the CAO oscillator 503 will oscillate with maximum magnitude at steady-state when $|\Omega| \times\left|\theta_{r}-\xi_{0}\right|$ is minimum. 

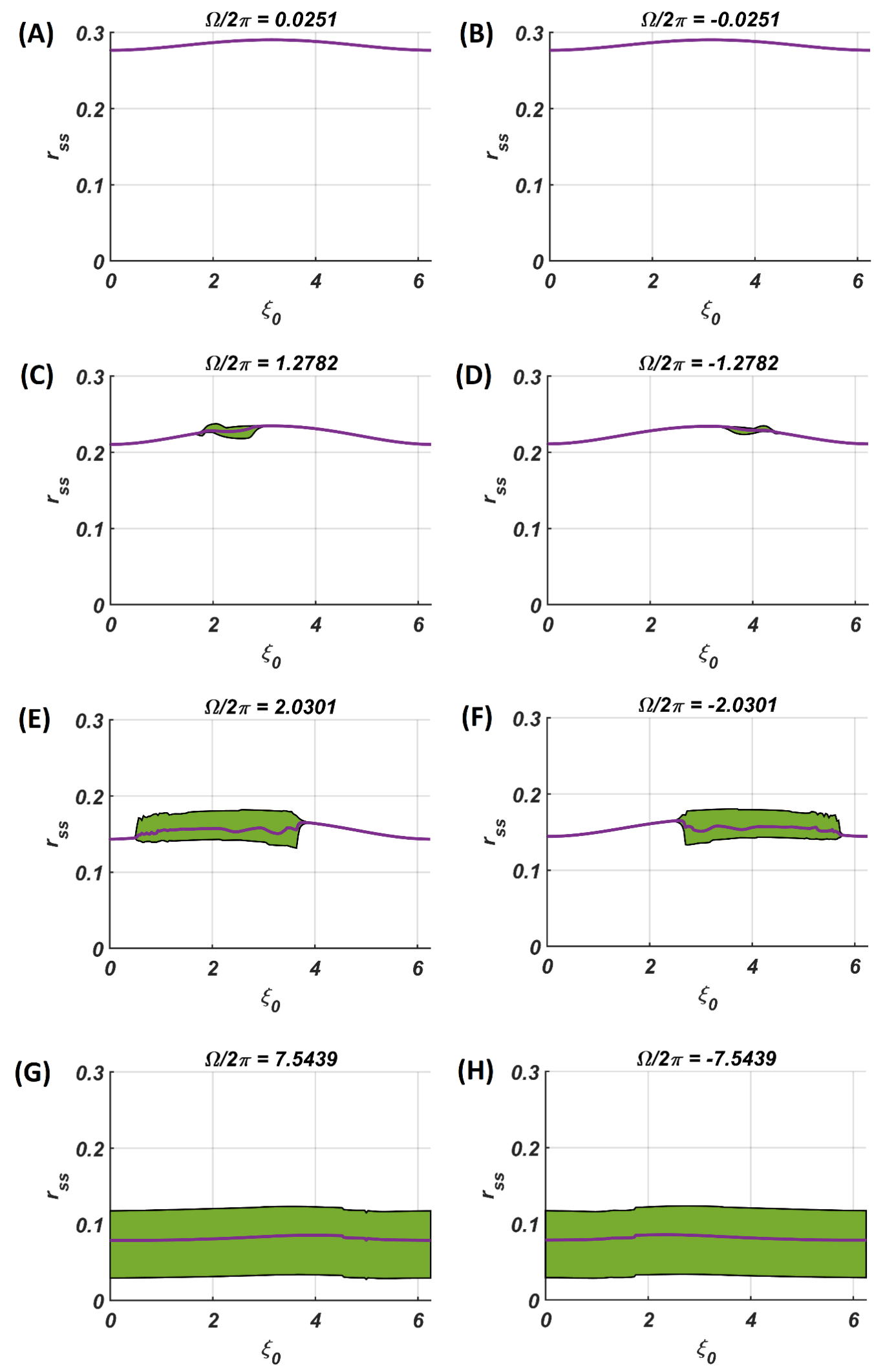

505 Figure 8: The steady-state magnitude of oscillation of the CAO oscillator with the identical pair of 506 coupled CAO oscillator and SRO as provided in the fig. 6 but for a symmetrical distinct value of the 
relative frequencies of the external input signal w.r.t the natural frequency of the CAO oscillator $\omega=$

$5082 \pi \times 60$, cross section of which is drawn in yellow lines in fig. 6 . The green region depicts the variance of steady state magnitude of oscillation w.r.t the mean value, plotted in purple.

510 Considering that the $\mathrm{CAO}$ oscillator is operating in the entrainment regime under the $1^{\text {st }}$ condition (eqn.

511 18a) and gets entrained to $I(t)$, the magnitude and the phase offset of oscillation at steady state is

512 analytically derived in Appendix-4. As the Hopf oscillator always maintains the same phase offset as

513 the phase offset of the complex sinusoidal input signal, the phase offset of the CAO oscillator becomes

514 the phase offset of the resultant input $\left(\operatorname{angle}\left(\bar{F}_{n e t}\right)\right)$, obtained by combining the external input, and

515 the input from the SRO through the complex power coupling connection (elaborated in Appendix-4).

$$
\bar{F}_{n e t}=\varepsilon F e^{i \xi_{0}}+A_{r} \frac{\mu_{r}}{\left|\beta_{1 r}\right|} e^{\frac{\omega_{0}}{2 \omega_{r}}} e^{i\left(\theta_{r}+\emptyset_{r}(0) \frac{\omega_{0}}{\omega_{r}}\right)}
$$

517 The first term on the right-hand side is the phasor of the external input $(I(t))$ through afferent weight,

518 and the second term is the phasor of the input from the SRO through modified power coupling. Note 519 that the magnitude of the resultant input after constructive/destructive interference, $\left|\bar{F}_{\text {net }}\right|$, determines

520 the magnitude of oscillation of the CAO oscillator at steady state. Fig-10C numerically verifies that 521 the CAO oscillator attains the analytically derived solutions in Appendix-4.

(A)

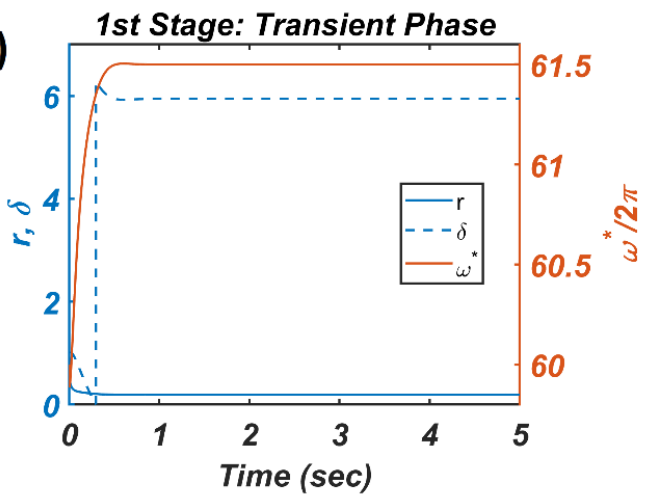

(C)

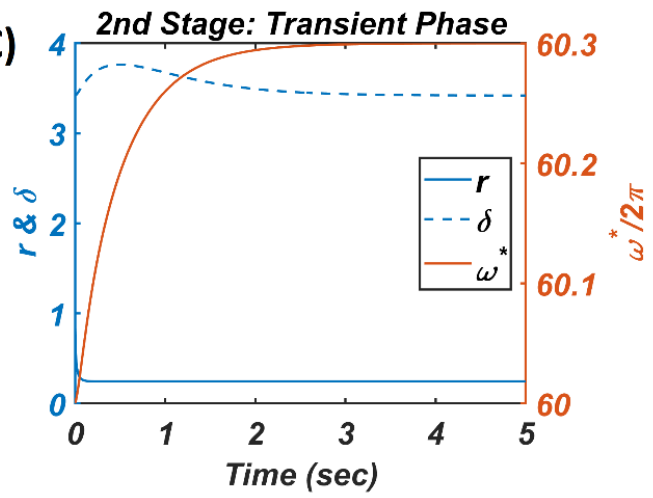

(B)

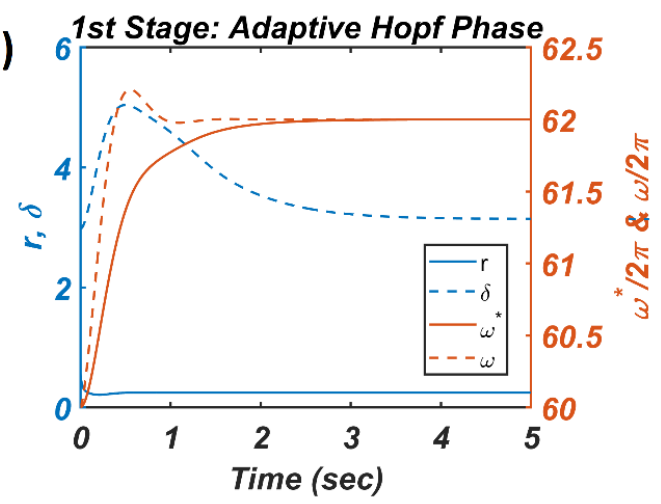

(D)

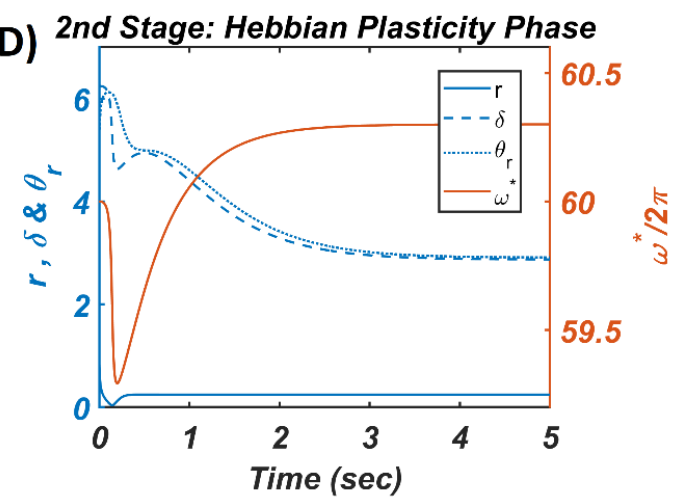


523 Figure 9: The figure depicts the response of the CAO oscillator in terms of its magnitude of oscillation

$524(r)$, phase offset $(\delta)$, entrainment $\left(\omega^{*}\right)$ during all the four phases of the $1^{\text {st }}$ and $2^{\text {nd }}$ stages of training 525 along with the parameters to be trained $\left(\omega\right.$ and $\theta_{r}$ ) and the other fixed parameters $\mu=1, \beta_{1}=150$, $526 \varepsilon=2, I_{0}=1, \mu_{r}=1, \beta_{1 r}=10, \omega_{r}=2 \pi \times 60.5$. (A) During the transient phase of the $1^{\text {st }}$ stage of 527 training the single unit is simulated under the entrainment regime of the CAO oscillator with the 528 additional parameters: $\omega_{0}=2 \pi \times 61.5, \xi_{0}=\frac{\pi}{4}, \theta_{r}=\pi, \tau \omega=8 \times 10^{-4}, A_{r}=10^{-5}$. It can be verified 529 that at steady-state $r$ and $\delta$ attain the solution provided by eqn. A1.8 and the value $\xi_{0}+\sin ^{-1} \frac{\Omega r_{S S}}{\varepsilon I_{0}}$ 530 respectively. (B) For the adaptive Hopf phase of the $1^{\text {st }}$ stage of training the single unit is simulated 531 with the additional parameters: $\omega(0)=2 \pi \times 60, \omega_{0}=2 \pi \times 62, \xi_{0}=\pi, \theta_{r}=\pi, \tau_{\omega}=0.5, A_{r}=$

$53210^{-5}, \eta_{\omega}=50$. It can be verified that at steady state $\delta$ attains $\xi_{0}$ and $\omega$ learns $\omega_{o}$. (C) The single unit 533 is simulated with the following set of additional parameters during the transient phase of the $2^{\text {nd }}$ stage 534 of training: $\omega_{0}=2 \pi \times 60.3, \xi_{0}=3.6773, \tau_{\omega}=0.5, A_{r}=0.1$. The steady-state values of $r$ depends 535 on $\left|\bar{F}_{\text {net }}\right|$ whereas the steady-state value of $\delta$ is same as $\arg \left(\bar{F}_{n e t}\right)$. (D) For the Hebbian plasticity 536 phase of $2^{\text {nd }}$ stage of training the single unit is simulated with the additional parameters: $\omega_{0}=$ $5372 \pi \times 60.3, \xi_{0}=\pi, \tau_{\omega}=0.5, A_{r}=10^{-5}, \eta_{\theta}=10^{-5}$. It can be verified that $\theta_{r}$ learns $\xi_{0}+$ $538 \sin ^{-1}\left(\frac{\Omega r_{S S}}{\varepsilon I_{0}}\right)$ which is same as the steady-state value of $\delta$.

539 The effect of $\mu, \beta_{1}$ and $\varepsilon I_{0}$ on the entrainment width $(\Delta \omega)$ and the typical transient time $\left(T_{t}\right)$ of the $540 \mathrm{CAO}$ oscillator for the $2^{\text {nd }}$ condition (eqn.18b), is further verified numerically. From fig. A2.2 in 541 Appendix-2 it can be verified that $\mu$ has to be small for wider entrainment regime, i.e., higher values 542 of $\mu$ causes shrinking of the entrainment regime. On the contrary $\varepsilon$ and $\beta_{1}$ have nearly the same effect 543 on the width of the entrainment regime: entrainment regime broadens as $\varepsilon$ and $\beta_{1}$ values are increased. 544 Since the transient dynamics determines the amount of time the network takes to settle down so that 545 the oscillator with the highest resonant response can be chosen, it is necessary to understand the effect 546 of these parameters on the transient dynamics. It is quite intuitive that $\mu$ and $\beta_{1}$ have a shrinking effect 547 on the transient period as it causes a much steeper basin of attraction around the steady state solution. 548 Figs. A2.2D and A2.2F show that increasing the magnitude of the scalar afferent weight, $\varepsilon$, shortens 549 the transient period; this is natural because a stronger input pushes the oscillator output to settle down 550 faster. Ideally, the model requires a broad entrainment regime with a short transient period. 


\section{Network level results:}

\section{First stage of training: training frequency}

554 We may recall from the previous section that the objective of the $1^{\text {st }}$ stage of training is to train the 555 frequencies of the CAO oscillators. To this end, only the external input signals are considered, and the

556 inputs from SRO are ignored (i.e., $A_{r} \ll \varepsilon$ in eqns. 9, 10,11). Initially the input set $U$ is constructed by 557 sampling $N_{f}$ number of intrinsic frequencies $\left(f_{o p}=\frac{\omega_{o p}}{2 \pi}\right)$ from a uniform distribution over the range 558 of 55 to $65 \mathrm{~Hz}$, and sampling $N_{\xi}$ number of phase offset angles $\left(\xi_{0 p}\right)$ from a uniform distribution over $559[0,2 \pi)$. Combining these sampled intrinsic frequencies and phase offsets, $N=N_{f} \times N_{\xi}$ number of 560 complex sinusoidal signal patterns $\left(I_{p}(t)=I_{0} e^{i\left(\omega_{0 p} t+\xi_{0 p}\right)}\right)$ are generated. After $I_{p}(t)$ chosen randomly 561 from $U$, the input patterns are presented one at a time to all the oscillators in CAO. Note that, in this 562 case, the winning CAO oscillator depends only on the condition $\min _{i j}\left|\omega_{i j}-\omega_{0 p}\right|$, and not on the phase 563 offset, $\xi_{0 p}$, since $A_{r} \ll \varepsilon I_{0}$.

564 For each presentation of the input, the winner oscillator and the oscillators in its neighbourhood, adjust 565 their 'preferred frequency' closer to the input frequency, $\omega_{0 p}$, following the adaptive Hopf learning rule of eqn. 16. The preferred frequency of the oscillators in the neighbourhood of the winner oscillator gets closer to $\omega_{0 p}$ compared to the oscillators near the periphery of the adaptive neighbourhood because of the Gaussian neighbourhood function, $\eta_{\omega_{i j}^{m n}}^{m n}$. The transient response of the CAO oscillator of a

569 single unit in terms of its magnitude of oscillation $(r)$, phase offset $(\delta)$, entrainment $\left(\omega^{*}\right)$ during the 570 transient phase is plotted in fig. 9A, where the analytically derived solutions of $r$ and $\delta$ (refer to 571 Appendix-2) is verified numerically. Similarly, the dynamic response of the same CAO oscillator in 572 the given single unit in terms of $r, \delta, \omega^{*}$ and $\omega$ during the adaptive Hopf phase is presented in fig. 9B, 573 where the analytically derived solutions of $r, \delta$ and $\omega$ (refer to Appendix-3) is verified numerically. 
(A)

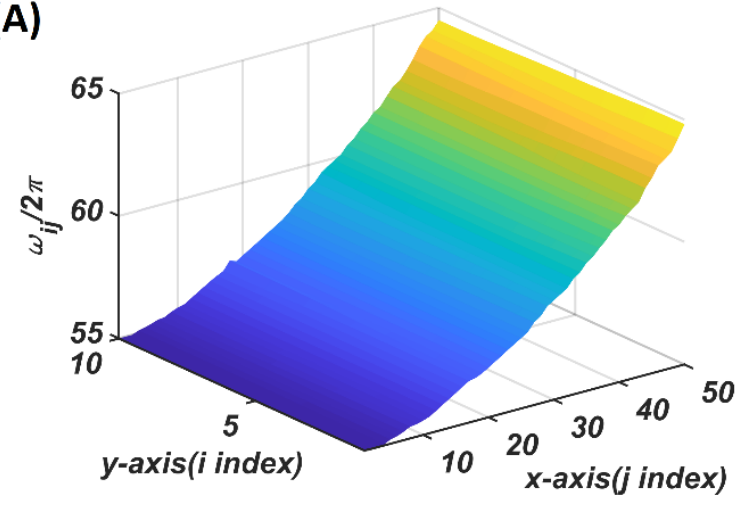

(C)

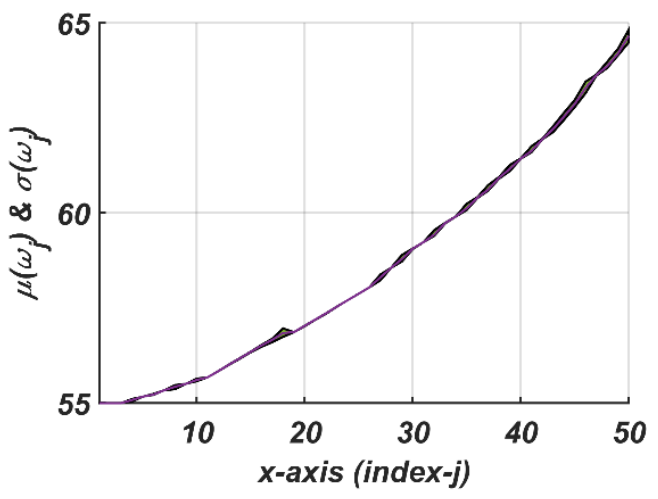

(B)

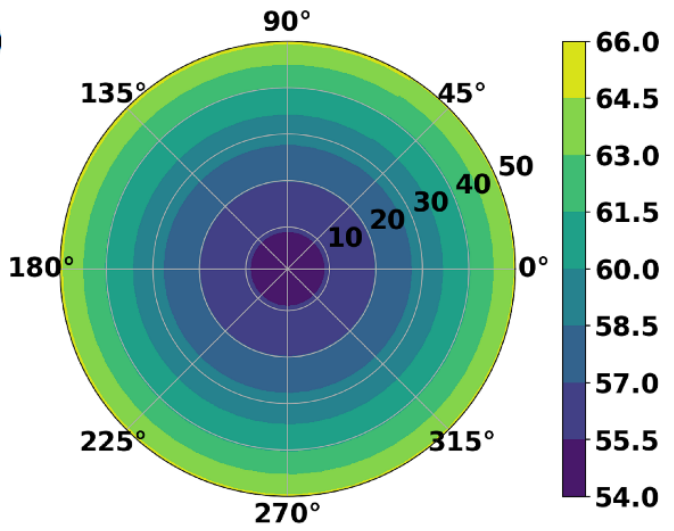

(D)

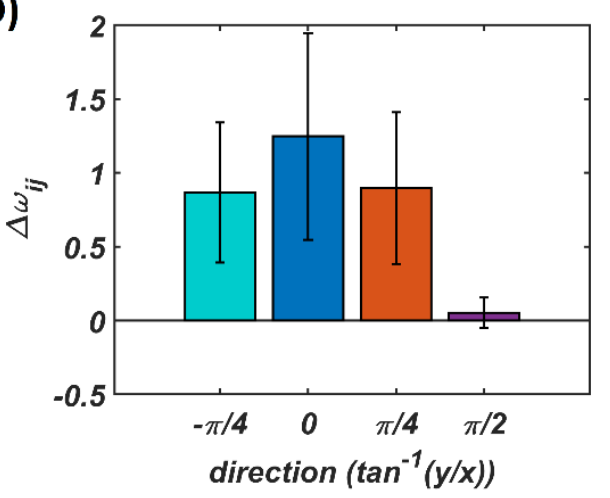

575 Figure 10: The natural frequency of oscillation, $\omega_{i j}$, of the oscillators in the CAO after self-

576 organization represented in Cartesian (A) and polar (B) organization. (C) shows mean and the variance of the natural frequencies along $\mathrm{x}$-axis after training. (D) provides a quantitative understanding of the

578 slope of self-organized $\omega_{i j}$ s along the x-axis, y-axis, and the axes $\frac{\pi}{4}$ and $-\frac{\pi}{4}$ inclined w.r.t the x-axis.

580 The natural frequencies of the CAO oscillators are initialized from a uniform random distribution confined to the interval $2 \pi[55,65]$. The natural frequency of the reference oscillator is set to the central

582 frequency $(2 \pi 60)$ of the given frequency band so that there is a symmetrical influence by the reference oscillator on CAO oscillators. The size of the CAO $\left(N_{r}, N_{c}\right)=(10,50)$, where $N_{r}$ is the number of rows and $N_{c}$ is the number of columns. The adaptive neighbourhood is defined by immediate proximity rather than the physical proximity, i.e., a neighbourhood size of $d_{W}=2$ means the immediate 2 oscillators on every side of the winner oscillator including the oscillators situated diagonally. 
589 column index but remains almost invariant along the orthogonal (row) direction. This emergent

590 tonotopic organization is the key feature of a self-organizing map. The parameter values defining the

591 network architecture, the data set and the training are given in Table-1 and Table-2.

\begin{tabular}{|cccl|}
$\begin{array}{l}\text { NETWORK } \\
\text { PARAMETERS }\end{array}$ & VALUES & $\begin{array}{l}\text { TRAINING } \\
\text { SET }\end{array}$ & VALUES \\
\hline$N_{x}$ & 50 & $U_{\omega}$ & $\omega_{0 p} \sim U(55 \times 2 \pi, 65 \times 2 \pi)$ \\
\hline$N_{y}$ & 10 & $U_{\xi}$ & $\xi_{0 p} \sim U(0,2 \pi)$ \\
\hline$\varepsilon$ & 2 & $N_{\omega}$ & 60 \\
\hline $\mathrm{dt}$ & $0.1 \mathrm{~ms}$ & $N_{\xi}$ & 10 \\
\hline$A_{i j}^{r}$ & $10^{-5}$ & $N=N_{\omega} N_{\xi}$ & 600 \\
\hline$\omega_{r}$ & $60 \times 2 \pi$ & & \\
\hline$\mu$ & 1 & \\
\hline$\mu_{r}$ & 1 & \\
\hline$\beta_{1}$ & 150 & \\
\hline$\beta_{1 r}$ & 10 & \\
\hline
\end{tabular}

592 Table-1: The table summarizes the list of parameters defining the overall network architecture and the 593 intrinsic parameters as well as the defining parameters of the training data set.

\begin{tabular}{|cccc}
$\begin{array}{l}\text { 1st } \text { STAGE } \\
\text { PARAMETERS }\end{array}$ & VALUES & $\begin{array}{l}\text { 2 }^{\text {nd }} \text { STAGE } \\
\text { PARAMETERS }\end{array}$ & VALUES \\
\hline$n_{i t e r, \omega}$ & 500 & $n_{i t e r, \xi}$ & 300 \\
\hline$T_{s \omega}$ & $3 \mathrm{sec}$ & $T_{s \theta}$ & 3 \\
\hline$T_{t \omega}$ & $4 \mathrm{sec}$ & $T_{t \theta}$ & 4 \\
\hline
\end{tabular}




\begin{tabular}{|c|c|c|c|}
\hline$\eta_{\omega_{0}}$ & 0.2 & $\eta_{\theta_{\max }}, \eta_{\theta_{\min }}$ & $2 \times 10^{-6}, 10^{-7}$ \\
\hline$\sigma_{y \omega m}$ & 100 & $\sigma_{y \theta m}$ & 2 \\
\hline$\sigma_{x \omega m}$ & 4 & $\sigma_{x \theta m}$ & 2 \\
\hline$\sigma_{\sigma x \omega}$ & $500 n_{e p h, \omega}$ & $\sigma_{\sigma x \theta}$ & $\infty$ \\
\hline$\sigma_{\sigma y \omega}$ & $500 n_{e p h, \omega}$ & $\sigma_{\sigma y \theta}$ & $\infty$ \\
\hline$d_{r}$ & 3 & $d_{r}$ & 3 \\
\hline$d_{c}$ & 3 & $d_{c}$ & 3 \\
\hline$A_{i j}^{r}$ & $10^{-5}$ & $A_{i j}^{r}$ & $\begin{array}{l}0.1 \text { in transient phase and } \\
10^{-5} \text { in Hebbian plasticity } \\
\text { phase }\end{array}$ \\
\hline
\end{tabular}

594 Table-2: The table summarizes the list of parameters essential for the $1^{\text {st }}$ and the $2^{\text {nd }}$ stage of training.

\section{Second stage of training: training phase offset}

596 As described in fig. 5B the $2^{\text {nd }}$ stage of training commences with the tonotopically organized $\omega_{i j}$ 's and 597 seeks to train $\theta_{i j}^{r}$ 's. The frequency and the phase offset of the external input are sampled from the same 598 set $U$. During the transient phase $A_{i j}^{r}$ is typically set to about a tenth of $\varepsilon$ so that the entrainment to $I_{e}(t)$ 599 can occur. From fig. 9C it can be observed that entrainment is possible even if $A_{r}$ is comparable with $600 \varepsilon$. It can be assumed that the winner oscillator will be the one which is going to satisfy the condition $601 \max _{i j}\left(\left|\bar{F}_{i j}^{n e t}\right|\right)$, where $\bar{F}_{i j}^{\text {net }}$ is the resultant input to the oscillator at $(i, j)$ in CAO. $\left|\bar{F}_{i j}^{n e t}\right|$ is a result of 602 constructive or destructive interference, between the external input and the input from the SRO, 603 depending on the relative values of $\xi_{0}$ and $\theta_{r}$, a qualitative explanation of which is discussed in 604 methods section with fig. 4 and the analytical expression of which is presented by eqns. A4.3 and A4.4 605 in Appendix-4.

606 The duration of the transient phase $\left(T_{s \theta}\right)$ in the $2^{\text {nd }}$ stage, is typically the same as the duration of the 607 transient phase $\left(T_{s \omega}\right)$ of the $1^{\text {st }}$ stage of training. With the proper choice of the network parameters, 608 principally $\varepsilon, A_{i j}^{r}, \mu, \mu_{r}, \beta_{1}, \beta_{1 r}$, as given in Table-1 and 2, the duration of the transient period $\left(T_{s \theta}\right)$ 
609 turned out to be approximately 3 secs. The CAO oscillator whose parameters $\left(\omega_{i j}, \theta_{i j}^{r}\right)$ are closest to 610 the input signal parameters $\left(\omega_{0 p}, \xi_{0 p}\right)$ will be the winner.

611 In the following Hebbian learning phase the neighbourhood size $\left(d_{r} \times d_{c}\right)$ is dependent on the size of 612 the entrainment window as the Hopf oscillator can only have a stable phase offset when it operates 613 inside its entrainment regime. From fig. 9D it can be observed that when the angle of the complex 614 power coupling coefficient $\left(\theta_{i j}^{r}\right)$ is trained according to the complex Hebbian learning rule (eqn. 17) 615 under the $2^{\text {nd }}$ condition (eqn. $18 \mathrm{~b}$ ) and $\dot{A}=0$, it learns the phase offset of the CAO oscillator. The 616 transient response of the CAO oscillator of a single unit in terms of its magnitude of oscillation $(r)$, 617 phase offset $(\delta)$, entrainment $\left(\omega^{*}\right)$ during the transient phase is plotted in fig. $9 \mathrm{C}$, where the analytically 618 derived solutions of $r$ and $\delta$ (refer to Appendix-4) is verified numerically. Similarly, the dynamic 619 response of the same CAO oscillator along with the angle of the complex modified power coupling 620 coefficient in the given single unit in terms of $r, \delta, \omega^{*}$ and $\theta_{r}$ during the Hebbian plasticity phase is 621 presented in fig. 9D, where the analytically derived solutions of $r, \delta$ and $\omega$ (refer to Appendix-3) is 622 verified numerically. As $\omega_{i j}$ parameters are self-organised in a monotonically increasing fashion along 623 the $\mathrm{x}$-axis and remains almost invariant along the other orthogonal dimension, the span of the trainable 624 neighbourhood window along the $\mathrm{x}$-axis has to be chosen dependent on the entrainment width of the 625 cortical oscillators. 

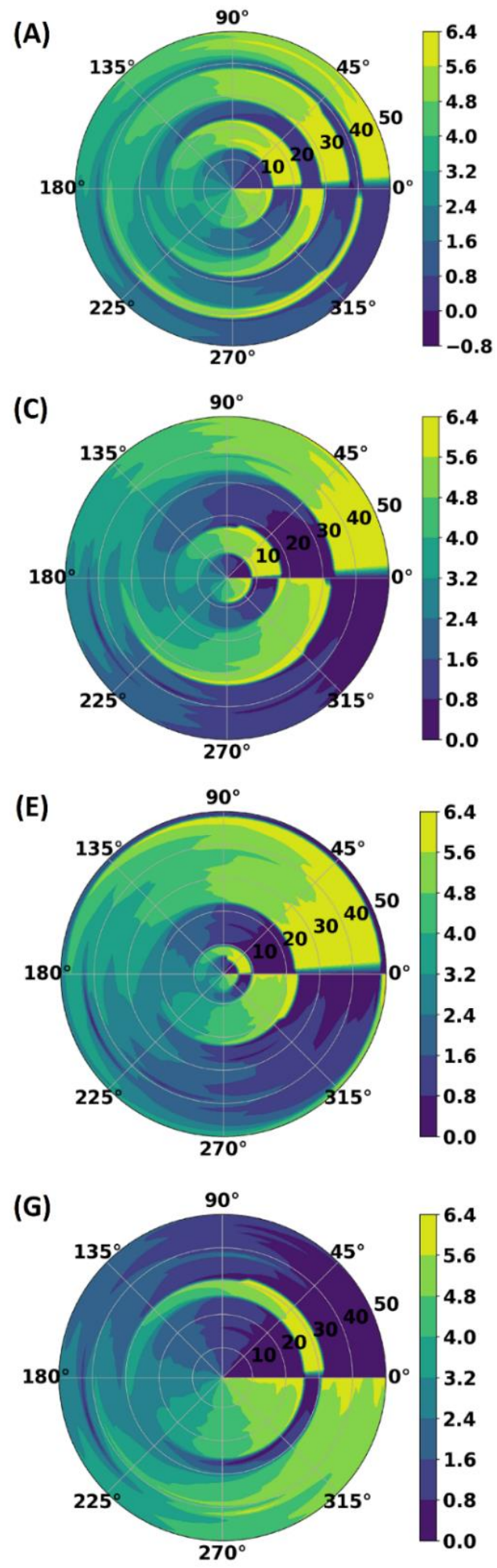

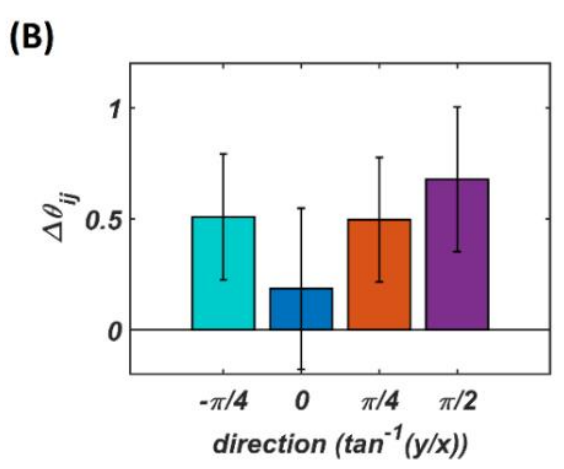

(D)

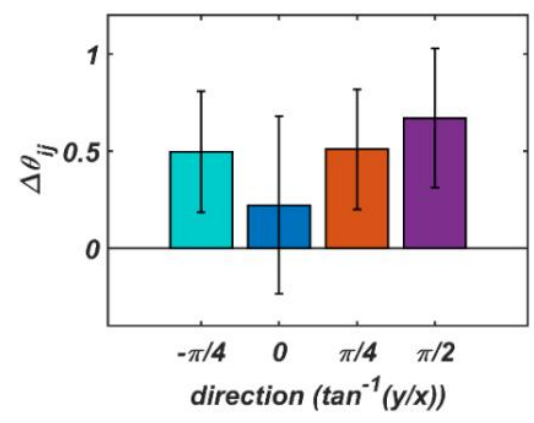

(F)

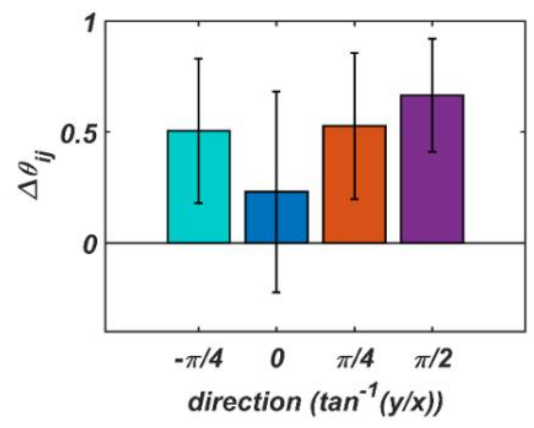

(H)

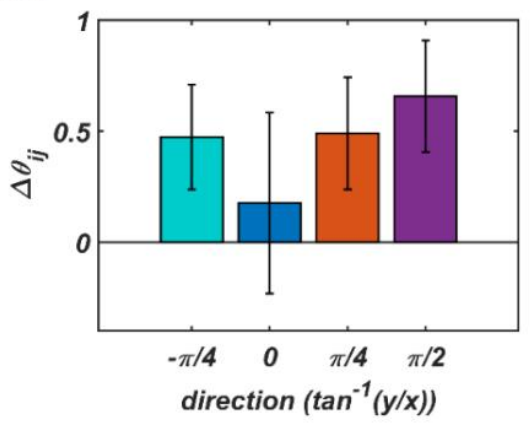

627 Figure 11: The self-organized $\theta_{i j}^{r}$ parameters after the $2^{\text {nd }}$ stage of training on four separate instances

628 starting with different randomly initialized $\theta_{i j}^{r}$ 's. The subplots $(\mathbf{B}),(\mathbf{D}),(\mathbf{F})$ and $(\mathbf{H})$ represent the mean

629 and the standard deviation of the gradients along the radial $(0)$, azimuth $\left(\frac{\pi}{2}\right)$, spirally diverging $\left(\frac{\pi}{4}\right)$ and 
630 converging $\left(-\frac{\pi}{4}\right)$ direction on a polar coordinate representation of the self-organized $\theta_{i j}^{r}$ s presented in 631 the subplots $(\mathbf{A}),(\mathbf{C}),(\mathbf{E})$ and $(\mathbf{F})$ respectively after the $2^{\text {nd }}$ stage of training.

632 With the parameters in Table 2, from fig. 11 it can be observed that the $\theta_{i j}^{r}$ parameters have self633 organized with a linear gradient along the y-axis but almost invariant along the x-axis. Fig. 11 634 represents four different instances of $2^{\text {nd }}$ stage of training with the pre-trained $\omega_{i j}$ parameters after the $6351^{\text {st }}$ stage of training as presented in fig. 10. The common features about all these four instances are: $636 \theta_{i j}^{r}$ s have either self-organised themselves in a linearly increasing or a decreasing fashion along the y637 axis or the azimuth direction in the polar coordinate system representation, it is unpredictable where 638 exactly along the x-axis the self-organized $\theta_{i j}^{r}$ s switch from an increasing to decreasing fashion and 639 vice-versa, the overall gradients of the self-organized $\theta_{i j}^{r}$ s along the 4 axes $(\mathrm{x}, \mathrm{y}$ and the axes inclined 640 at an angle $\frac{\pi}{4}$ and $-\frac{\pi}{4}$ w.r.t the $\mathrm{x}$-axis) for all these four instances are statistically similar. If the Gaussian 641 shape of the neighbourhood function of $\omega_{i j}, \eta_{\omega_{i j}}^{m n}$, defined by its standard deviations along x- and y642 axis $\left(\sigma_{x \omega}, \sigma_{y \omega}\right)$ is compared with the Gaussian shape of the neighbourhood function of $\theta_{i j}^{r}, \eta_{\theta_{i j}}^{m n}$ $643\left(\sigma_{x \theta}, \sigma_{y \theta}\right)$, the variances of $\eta_{\theta}^{m n}$ is much smaller than the variances of $\eta_{\omega_{i j}}^{m n}$, particularly along the y-

644 axis. Also, the distribution of $\eta_{\theta_{i j}}^{m n}$ has a similar spread along both of the orthogonal axes. Distribution

645 of $\omega_{i j}$ s from fig. 10D compared to the distributions of $\theta_{i j}^{r}$ s presented in fig. 11B, D, E and F confirms 646 that they self-organize along orthogonal directions.

\section{Testing:}

648 During the testing phase of the OTSOM model, the conventional power coupling is used from the SRO 649 to the CAO oscillator. The dynamics of the model during the testing phase is described as below:

$$
r_{\imath j}=\left(\mu-\beta_{1} r_{i j}^{2}\right) r_{i j}+A_{i j}^{r} r_{r}^{\frac{\omega_{i j}}{\omega_{r}}} \cos \omega_{i j}\left(\frac{\theta_{i j}^{r}}{\omega_{i j}}+\frac{\emptyset_{r}}{\omega_{r}}-\frac{\emptyset_{i j}}{\omega_{i j}}\right)+\varepsilon \operatorname{real}\left(I(t) e^{-\emptyset_{i j}}\right)
$$

$$
\dot{\emptyset}_{l j}=\omega_{i j}+A_{i j}^{r} \frac{r_{r}^{\frac{\omega_{i j}}{\omega_{r}}}}{r_{i j}} \sin \omega_{i j}\left(\frac{\theta_{i j}^{r}}{\omega_{i j}}+\frac{\emptyset_{r}}{\omega_{r}}-\frac{\emptyset_{i j}}{\omega_{i j}}\right)+\frac{\varepsilon}{r_{i j}} \operatorname{imag}\left(I(t) e^{-\emptyset_{i j}}\right)
$$

$$
\dot{r}_{r}=\left(\mu_{r}-\beta_{1 r} r_{r}^{2}\right) r_{r}
$$

$$
\dot{\phi}_{r}=\omega_{r}
$$


654 It can be observed that the ratio of the natural frequencies of the CAO oscillators w.r.t the reference 655 oscillator is raised to power of the complex activation of the reference oscillator instead of the actual 656 frequencies of the CAO oscillator w.r.t the reference oscillator. For the moment, real sinusoidal signals 657 or linear combination of multiple real sinusoidal signals are used as external input $(I(t))$. At first, we 658 are going to analyse the steady state response of a single CAO oscillator by considering the DC 659 component of the magnitude of oscillation of the CAO oscillator numerically. The DC component of 660 the steady state magnitude of oscillation is extracted using a third order low pass filter with a cut off 661 frequency of $0.01 \mathrm{~Hz}$. From fig. 12 it can be observed that the DC component of the steady state 662 magnitude of oscillation preserves the encoding ability of all the characteristic components of the 663 sinusoidal input signal. The resonance exhibited w.r.t the frequency of real sinusoidal external input is 664 similar to the case of complex sinusoidal external input as observed in figs. 13A and D. Whereas the 665 resonance exhibited w.r.t the phase offset of real sinusoidal external input is confined to a very narrow 666 band-width of the frequency of real sinusoidal external input w.r.t the natural frequency of the CAO 667 oscillator (figs. 13B, 13E).

668 The response of the OTSOM model is tested with three types of signals: periodic real sinusoidal signal 669 (fig. 13A and 13B), quasi-periodic signal which is a combination two proximal frequency components 670 (fig, 13C and 13D) given in the caption of fig. 13 and an aperiodic signal such as an 671 Electroencephalograph (EEG) signal as plotted in fig. 14A with its characterizing power spectrum 672 plotted in fig. 14B. The EEG signal was collected during mind wondering task with a sampling rate of $6731024 \mathrm{~Hz}$ (Grandchamp et al., 2014). The characterizing $\omega_{i j}$ and $\theta_{i j}^{r}$ parameters during the testing phase 674 is illustrated in fig. 10 and $12 \mathrm{C}$ respectively. It can be observed from fig. 13C and 13D that the 675 representation of the frequency $55.669 \mathrm{~Hz}$ is broader w.r.t the frequency $61.426 \mathrm{~Hz}$, which is because 676 of the slope of $\omega_{i j} \mathrm{~s}$ along the x-axis at around $55.669 \mathrm{~Hz}$ is lesser than the slope at around $61.426 \mathrm{~Hz}$. 
(A)

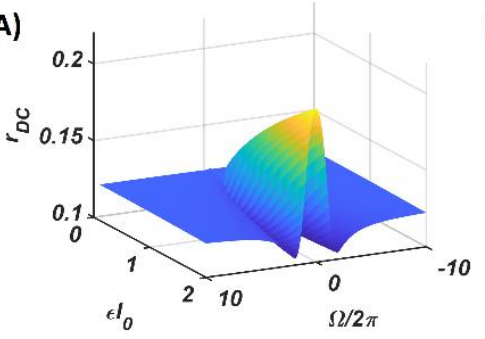

(D)

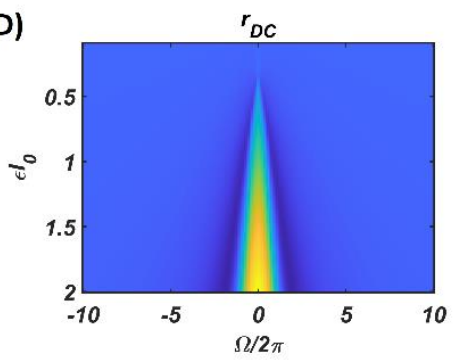

(B)

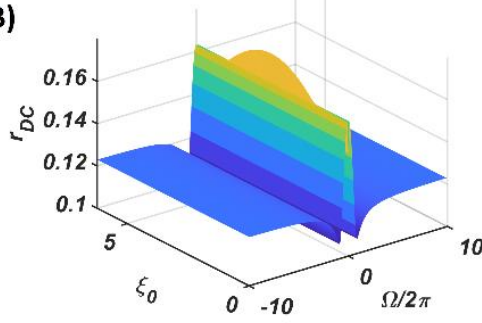

(E)

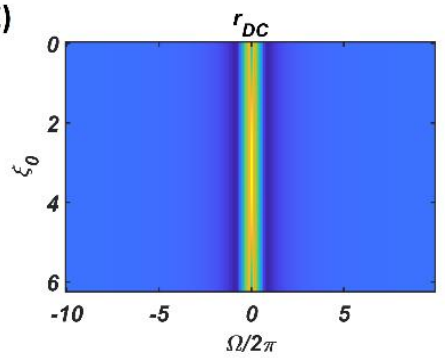

(C)

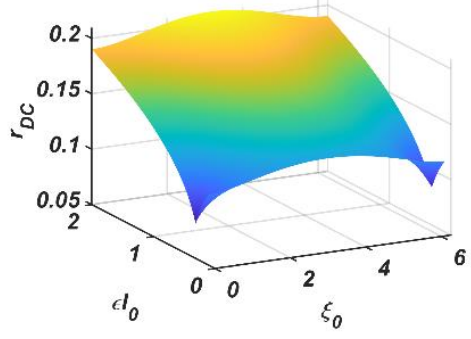

(F)

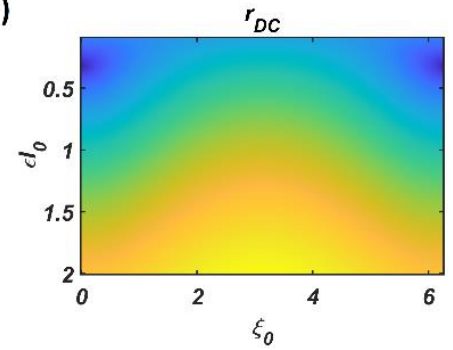

678 Figure 12: The DC response of the CAO oscillator w.r.t the three properties of the external input 679 signal; amplitude $\left(\varepsilon I_{0}\right)$, frequency $\left(\omega_{0}\right)$ and phase offset $\left(\xi_{0}\right)$. For all these simulations the common 680 parameters are: $\mu=1, \beta_{1}=-150, \omega=2 \pi \times 60, F=1, \mu_{r}=1, \beta_{1 r}=-10, \omega_{r}=2 \pi \times 60, A_{r}=$ $6810.5, \theta_{r}=\pi$. For the plots $\omega_{0}$ is varied from $2 \pi \times 50$ to $2 \pi \times 70, \xi_{0}$ is varied from 0 to $2 \pi$, $\varepsilon$ is varied 682 from 0.1 to 2. For the plots in the left column $\xi_{0}$ is kept fixed at a value of $\pi$. For the plots in the 683 middle column $\varepsilon$ is kept fixed at a value of 1 . For the plots in the left column $\omega_{0}$ is kept fixed at a value 684 of $2 \pi \times 60$. 

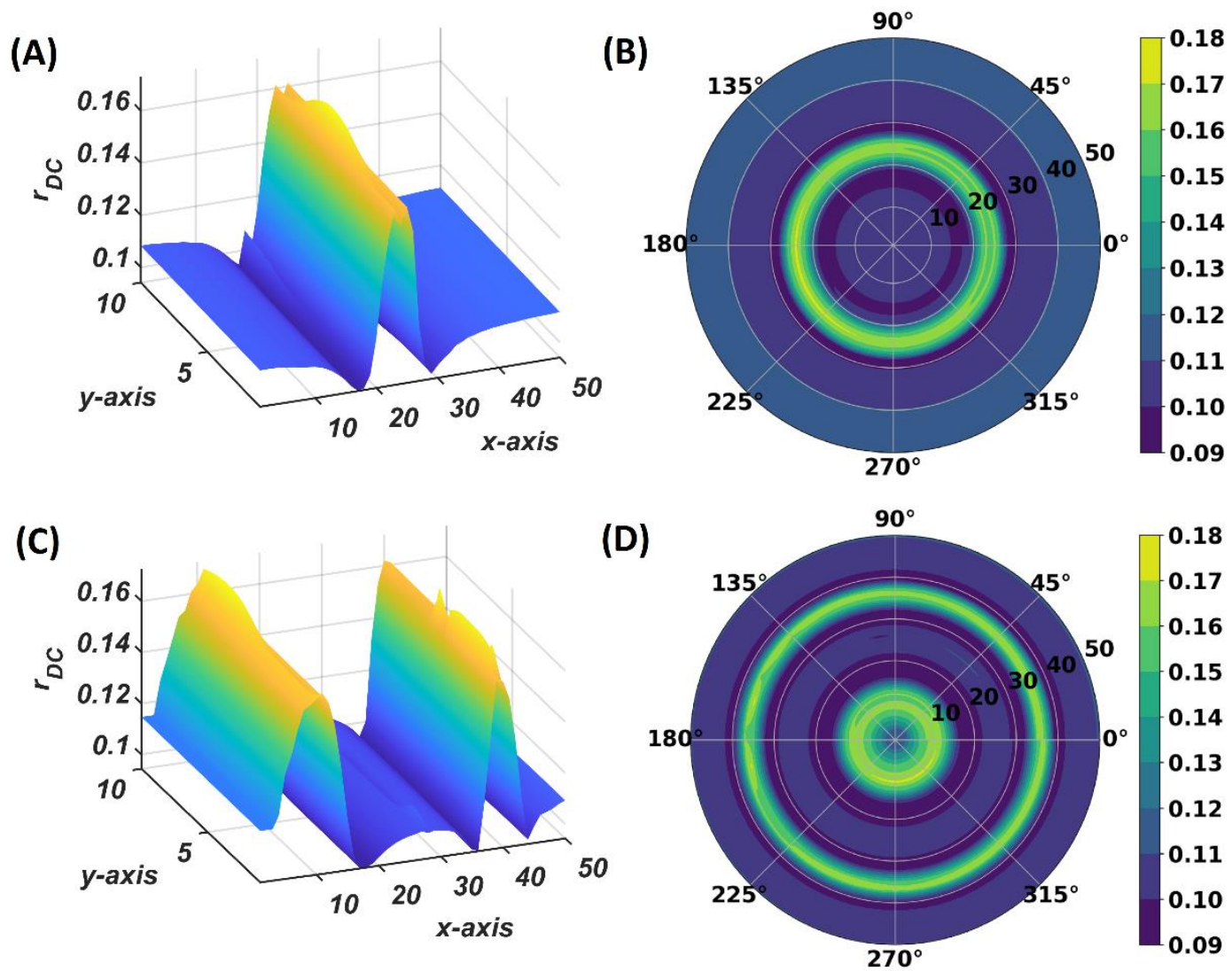

686 Figure 13: The subplots (A) and (B) depicts the response of the OTSOM model when the external 687 input signal is: $I(t)=\cos (2 \pi \times 57.7029+\pi)$. The subplots $(\mathbf{C})$ and $(\mathbf{D})$ depicts the response of the 688 OTSOM model when the external input signal is: $I(t)=\cos \left(2 \pi \times 61.426+\frac{3 \pi}{2}\right)+\cos (2 \pi \times$ $\left.68955.669+\frac{\pi}{2}\right)$. The $\omega_{i j}{ }^{\prime} s$ of the CAO oscillators and the $\theta_{i j}^{r}$ 's are adopted from the training stage of the 690 OTSOM model as depicted in the fig. 10 and fig. 11C. The other parameters of the model are mostly 691 preserved as used in the training session of the model: $\mu=1, \beta_{1}=150, F=1, \mu_{r}=1, \beta_{1 r}=1, \omega_{r}=$ $6922 \pi \times 60, A_{r}=0.1$. 
(A)

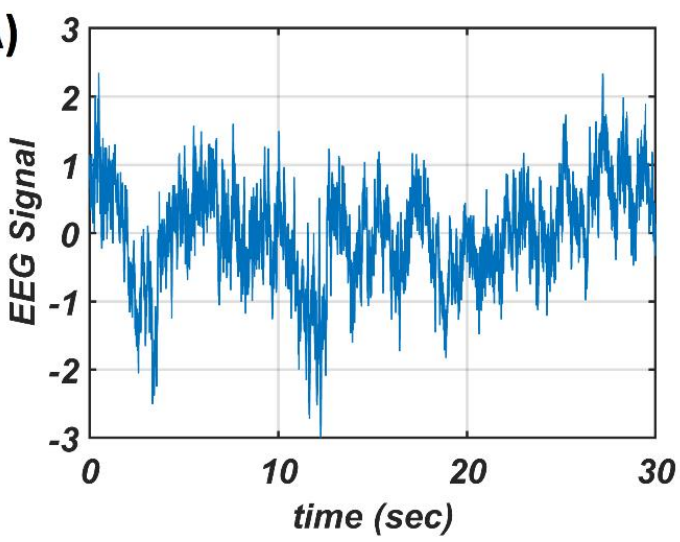

(C)

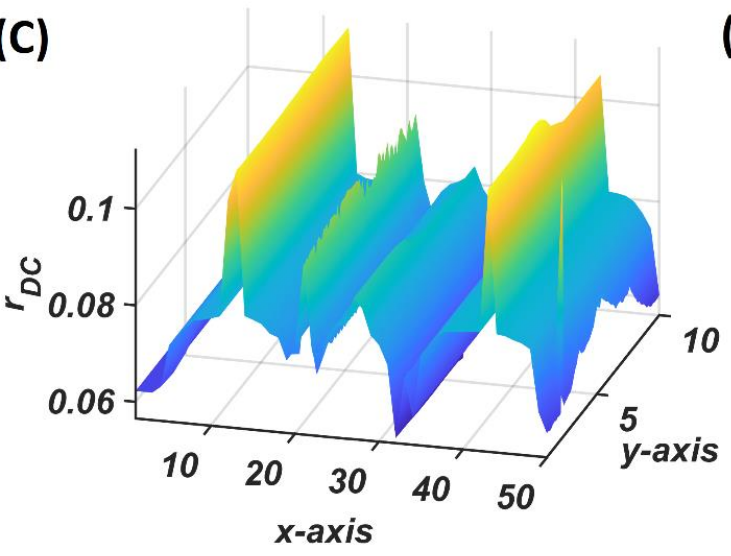

693

694 Figure 14: The subplots (C) and (D) illustrates the response of the OTSOM model when an arbitrary 695

696

697

698

699

700

701

702

703

704 aperiodic signal such as the EEG signal plotted in (A) characterized by its power spectrum plotted in (B) is presented as external input signal.

\section{Discussion}

Tonotopic map refers to a map of tones or individual frequencies often found in auditory cortices of mammals. Optimal response at a specific frequency is characteristic of resonance. Based on this insight, we designed a tonotopic map model based on nonlinear oscillators capable of exhibiting resonance. We present a model of a tonotopic map, which consists of an array of Hopf oscillators, labeled as CAO. The map is trained on complex sinusoidal stimuli such that the frequency is mapped onto to the columns, and phase is mapped onto the rows. (The phase of the input signal is defined with reference to a reference oscillator labeled as SRO.) In other words, when a complex sinusoid with a given frequency and phase is presented as input stimulus, the oscillator at a specific row and column, whose frequency and phase are the closest to the input parameters, responds with the highest amplitude.
(B)

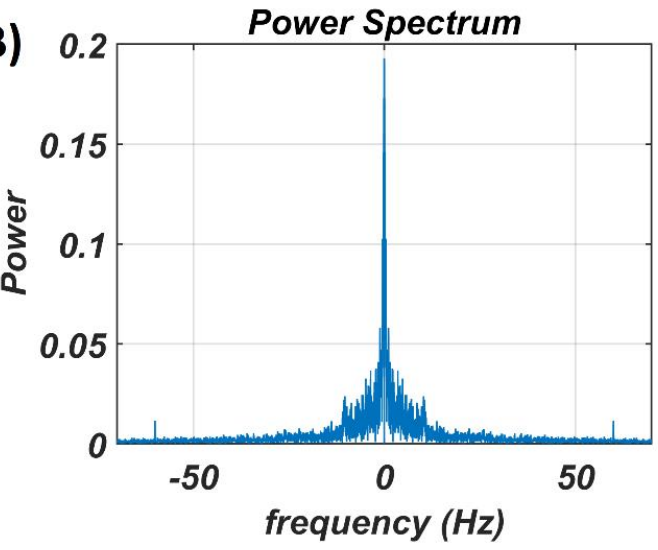

(D)

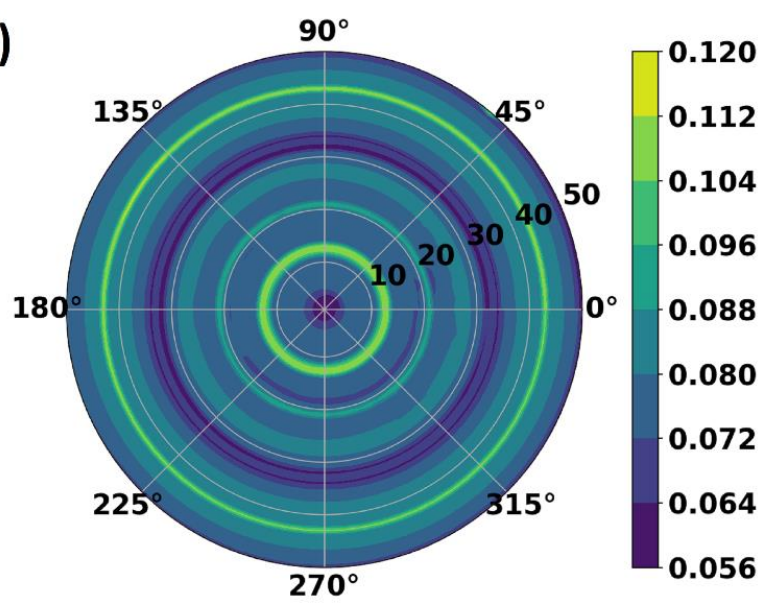


708 Existing computational models of tonotopic map do not attempt to model the underlying oscillation or

709 the associated resonance in modeling tuned responses to pure tones. In the tonotopic model of (Ritter 710 et al., 1992), which is based on a SOM model, frequencies are modeled as explicit parameters defined 711 out of the context of the underlying oscillatory process. The model was able to achieve an ordered map 712 of frequencies, with greater areas of the map differentially allotted to dominant frequencies in the input.

713 However, the model was not able to capture any other temporal aspects of the input signal, since no 714 signal was explicitly modeled. Another tonotopic map model described by Palakal et al., 1995 modeled 715 the distribution of both frequency and time delay. But here too, these parameters are described as 716 independent parameters, taken out of the context of the underlying temporal process. In this regard, the 717 proposed tonotopic model based on oscillators and resonance represents a significant step forward.

718 A previous model (Biswas et al., 2021) that shows how a network of Hopf oscillators can be trained to 719 learn arbitrary aperiodic signals was developed further to create the proposed tonotopic map model.

720 To this end two improvements had to be made to the previous model:

721 a) a key element of (Biswas et al., 2021) is the concept of power coupling that achieves a stable 722 (normalized) phase relationship between a pair of oscillators with arbitrary intrinsic frequencies. This 723 scheme had to be modified in the proposed model since it must allow mixed forms of coupling combining power coupling with ordinary real coupling.

725 b) in the proposed model, oscillators must exhibit tuned responses not only to frequency but also to 726 phase. In order to define a phase offset of the input signal, we introduced a reference oscillator (SRO) 727 that projects to all the oscillators in the map.

728 The functional unit of OTSOM model is a single CAO oscillator that receives input from the external 729 input and the SRO. We performed qualitative and the quantitative analysis of this unit under two 730 conditions:

731 1) Magnitude of the SRO input is negligible compared to the external input.

732 2) Magnitude of the SRO input is comparable to the external input.

733 The magnitude of the input from the reference oscillator is $A_{r} r_{r}^{\frac{\omega^{*}}{\omega_{r}^{*}}}$, where the steady state value of the 734 magnitude of oscillation of the SRO depends on $\mu_{r}$ and $\beta_{1 r}$. As the SRO operates in the supercritical

735 Hopf regime both $\mu_{r}$ and $\beta_{1 r}$ are positive and the steady state magnitude of oscillation is $\sqrt{\frac{\mu_{r}}{\beta_{1 r}}}$. With 
this simple setup we have observed that the CAO oscillator can encode not only the frequency of the complex sinusoidal input signal primarily inside the entrainment regime, but also the phase offset of the input signal.

The entrainment regime of a canonical Hopf oscillator is previously analyzed by (Kim \& Large, 2015) in terms of analyzing steady-state dynamical characteristics on $r-\psi$ plane. As $\psi$ is the angular difference between the oscillator and the external input signal, when the system exhibits stable fixed point $\left(\Delta>0, T^{2}-4 \Delta>0, T<0\right)$ or stable spiral behaviour $\left(\Delta>0, T^{2}-4 \Delta<0, T<0\right)$ it can be interpreted that the system is entrained. When the relative phase of the oscillator w.r.t the input signal reaches a steady state value, it essentially means the actual frequency of oscillation of the oscillator is adapted from its natural frequency of oscillation to the frequency of the driving signal. Kim and Large (Kim \& Large, 2015) have analyzed the effect of the strength of the driving signal on its entrainment characteristics by mapping the nature of the steady-state solution on the $\varepsilon F$ vs $\Omega$ space. There are five possible steady state solutions exhibited by four regimes of canonical Hopf oscillator defined by its intrinsic parameter values. These five steady state solutions are stable node, stable spiral, unstable node, unstable spiral and saddle point. When the Hopf oscillator operates in critical Hopf parameter regime $\left(\mu=0, \beta_{1}>0, \beta_{2}=0\right)$ it exhibits either stable node or stable spiral solution at steady-state, i.e., for any values of its intrinsic parameter $\beta_{1}$, the strength of the driving signal $(\varepsilon F)$ and $\Omega$ it is going to be entrained to the frequency of the driving signal. Therefore, it can be stated that the entrainment regime of the Hopf oscillator operating in the critical parameter regime is unbounded. In both of these cases the state of the system reaches the fixed point asymptotically, i.e., it takes forever for the oscillator to get entrained. Generally, a small neighbourhood around the fixed point is defined to declare the entrainment of the system. The Hopf oscillator operating in the supercritical parameter regime $(\mu>$ $0, \beta_{1}>0, \beta_{2}=0$ ) exhibits three steady-state solutions: stable fixed point, stable spiral and unstable spiral. Till the boundary to the unstable spiral solution the system exhibits entrainment.

The typical initial value of the variance $\eta_{\omega}$ has the property: $\sigma_{y \omega m} \gg \sigma_{x \omega m}$. Due to high variance along the column, the other oscillators in the same column as the winner tend to adapt to the feature of the presented input pattern at the same rate as the winner neuron, which ensures the low variability of the learnt natural frequency of the oscillator along $y$ - axis. An initial standard deviation of $\sigma_{y \omega m}=$ 100 and $\sigma_{x \omega m}=4$ is sufficient for the tonotopic organization to arise as presented in fig. 10. Although there is a lower bound for $\sigma_{y \omega m}$ depending on the number of oscillators along the $y$-direction, $N_{y}$, 
767 be interpreted that the lower bound on $\sigma_{y \omega m}$ should be proportional to $N_{y}$ as the greater the number of 768 oscillators per column, the lesser the adaptability rate of the oscillators at the boundaries of the adaptable neighbourhood along the column. However, a square neighbourhood window function is chosen for the simulation presented in this study, a rectangular window function is also feasible. A rectangular window function can be defined by $d_{r} \neq d_{c} . \sigma_{x \omega}$ and $\sigma_{y \omega}$ decrease in a Gaussian contour w.r.t time at a much slower time scale to model the effect of annealing. $\sigma_{x \omega}$ and $\sigma_{y \omega}$ are updated after every epoch, with a typical standard deviation on an iterative time scale of $500 n_{e p h, \omega}$.

A few aspects need to be elaborated about the $2^{\text {nd }}$ stage of training. The $\theta_{i j}^{r}$ s were failing to selforganizing themselves in a linearly increasing or decreasing fashion along the column when the CAO oscillators were placed on a 2-dimensional rectangular grid. To fix this issue, periodic boundary condition is introduced along the spatial dimension of the column i.e., the bottom row of the CAO is closest with an equidistant to both the top row as well as the second last row which is the motivation behind representing the $\mathrm{CAO}$ on a polar coordinate representation. Although this ensured that the $\theta_{i j}^{r} \mathrm{~s}$ self-organize themselves in a linearly increasing or decreasing manner in a given column, $\theta_{i j}^{r} \mathrm{~s}$ were slipping at a constant rate along the azimuth axis which can be observed in fig. 15A. To fix this problem the $\theta_{i j}^{r} \mathrm{~s}$ of the top most row of the CAO were fixed at $0^{\circ}$ angle and from fig. $15 \mathrm{~B}$ it can be observed that $\theta_{i j}^{r} \mathrm{~s}$ of a given column were stabilized with a linear organization. On the contrary, the $\omega_{i j} \mathrm{~s}$ are able to self-organize themselves without the aforementioned periodic boundary condition.
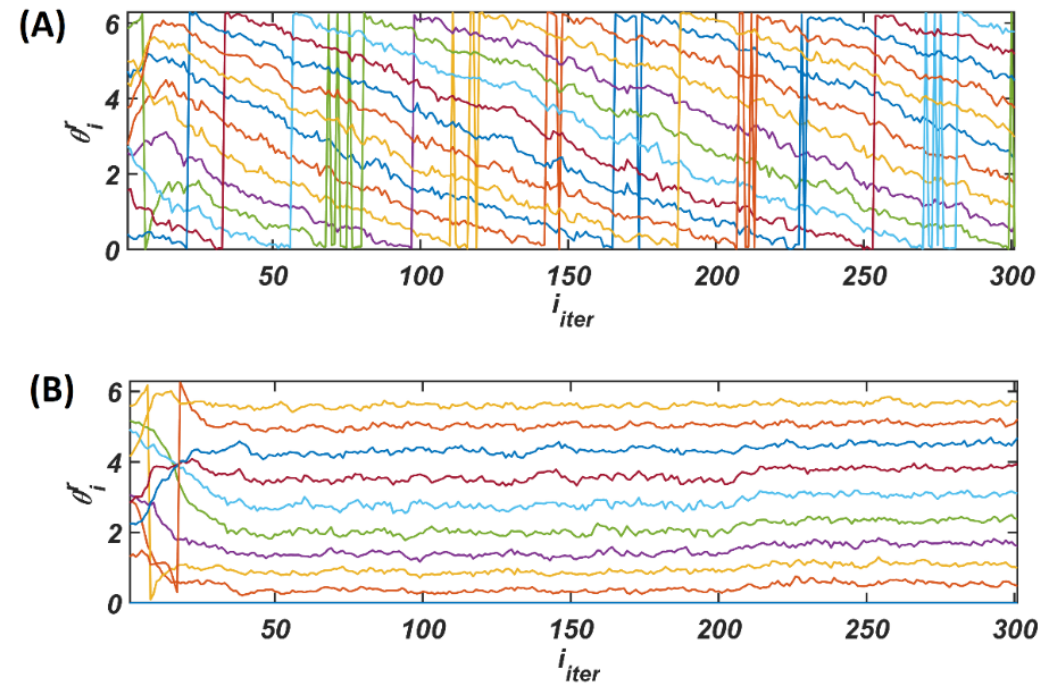

786 Fig15: (A) Evolution of the $\theta_{i j}^{r}$ s of a given column over multiple epochs in the $2^{\text {nd }}$ stage of training 787 with the periodic $\mathrm{CAO}$ along the dimension of $\mathrm{y}$-axis, (B) Self organization of $\theta_{i j}^{r}$ s of the same column 

fixed $\theta_{i j}^{r}$ s of the top most row with $0^{\circ}$ angle.

A Comparison with the conventional SOM: For conventional SOM model (Kohonen, 1998), the neuronal response is characterized by its linear or nonlinear activation function. When these rate coded neurons are a part of SOM framework, the afferent weights for a particular neuron are also considered to be an internal feature of the neurons, considering close proximity of these afferent synapses to the corresponding neurons. The key differences between the conventional SOM model and OTSOM are:

1) the afferent connection weights are fixed,

2) the input is time varying complex sinusoidal signal instead of a constant vector,

3) the neurons are limit cycle oscillators instead of rate-coded neurons with a static transfer function.

Although we have tested the model with complex sinusoidal input signals sampled from the frequency band from 55 to $65 \mathrm{~Hz}$, the bandwidth can be scaled up/down or shifted. The proposed model can be used to explain the tonotopic organization evolved in auditory cortex of mammals.

\section{$5 \quad$ Conflict of Interest}

803 The authors declare that the research was conducted in the absence of any commercial or financial 804 relationships that could be construed as a potential conflict of interest.

\section{Author Contributions}

806 DB and AT: hypothesis testing, conceptualization, theory development, numerical simulations, 807 investigation, methodology, and validation. DB and VC: visualization and writing - original draft. 808 VC: writing - review, editing, and supervision. All authors contributed to the article and approved 809 the submitted version.

\section{Data availability statement}

811 The original contributions presented in the study are included in the article/Supplementary Material,

812 further inquiries can be directed to the corresponding author/s.

\section{$813 \quad 8 \quad$ Funding}

814 The authors would like to thank MHRD, Govt. of India for the HTRA scholarship for Ph.D. students.

\section{Acknowledgments}

816 We acknowledge the support of fellow lab mate Sayan Ghosh for preprocessing the EEG signals 


\section{References}

818

819

820

821

822

823

824

825

826

827

828

829

830

831

832

833

834

835

836

837

838

839

840

841

842

843

844

845

846

847

848

849

850

851

852

Bates, M. E., Simmons, J. A., \& Zorikov, T. V. (2011). Bats use echo harmonic structure to distinguish their targets from background clutter. Science, 333(6042), 627-630. https://doi.org/10.1126/science.1202065

Bekesy, G. von. (1949). The vibration of the cochlear partition in anatomical preparations and in models of the inner ear. J Acoust Soc Am, 240(1948).

Biswas, D., Pallikkulath, S., \& Chakravarthy, V. S. (2021). A Complex-Valued Oscillatory Neural Network for Storage and Retrieval of Multidimensional Aperiodic Signals. Frontiers in Computational Neuroscience, 15(May), 1-24. https://doi.org/10.3389/fncom.2021.551111

Boynton, G. M., Stecker, G. C., Huber, E., Thomas, J. M., Saenz, M., \& Fine, I. (2015). Population receptive field estimates of human auditory cortex. NeuroImage, 206, 428-439. https://doi.org/10.1016/j.neuroimage.2014.10.060.Population

Clopton, B. M., Winfield, J. A., \& Flammino, F. J. (1974). TONOTOPIC ORGANIZATION: REVIEW AND ANALYSIS. Brain Research, 76, 1-20.

Ehret, G., \& Romand, R. (1996). The Central Auditory System (G. Ehret \& R. Romand (eds.)). Oxford University Press.

Farokhniaee, A., Almonte, F. V, Yelin, S., \& Large, E. W. (2020). Entrainment of Weakly Coupled Canonical Oscillators with Applications in Gradient Frequency Neural Networks Using Approximating Analytical Methods. Mathematics. https://doi.org/10.3390/math8081312

Frank Julicher, Andor, D., \& Duke, T. (2001). Physical basis of two-tone interference in hearing. Pnas.

Fredrickson-hemsing, L., Strimbu, C. E., Roongthumskul, Y., \& Bozovic, D. (2012). Dynamics of Freely Oscillating and Coupled Hair Cell Bundles under Mechanical Deflection. Biophysj, 102(8), 1785-1792. https://doi.org/10.1016/j.bpj.2012.03.017

Grandchamp, R., Braboszcz, C., \& Delorme, A. (2014). Oculometric variations during mind wandering. Frontiers in Psychology, 5(FEB), 1-10. https://doi.org/10.3389/fpsyg.2014.00031

Hadjikhani, N., Liu, A. K., Dale, A. M., Cavanagh, P., \& Tootell, R. B. H. (1998). Retinotopy and color sensitivity in human visual cortical area V8. Nature Neuroscience, 235-241.

Hubel, D. . H. ., \& Wiesel, T. . N. . (1959). RECEPTIVE FIELDS OF SINGLE NEURONES IN THE CAT' S STRIATE CORTEX. J. Physiol, 574-591.

IMIG, T. J., \& ADRIAN, H. O. (1977). BINAURAL COLUMNS IN THE PRIMARY FIELD (A1) OF CAT AUDITORY CORTEX. 138, 241-257. https://doi.org/https://doi.org/10.1016/00068993(77)90743-0

Kim, J. C., \& Large, E. W. (2015). Signal processing in periodically forced gradient frequency neural networks. Frontiers in Computational Neuroscience, 9(DEC), 1-14.

https://doi.org/10.3389/fncom.2015.00152 
853

854

855

856

857

858

859

860

861

862

863

864

865

866

867

868

869

870

871

872

873

874

875

876

877

878

879

880

881

882

883

884

885

886

887

888

Kohonen, T. (1988). An introduction to neural computing. Neural Networks, 1(1), 3-16. https://doi.org/10.1016/0893-6080(88)90020-2

Kohonen, T. (1990). The Self-Organizing Map. Proceedings of the IEEE, 78(9), 1464-1480. https://doi.org/10.1109/5.58325

Kohonen, T. (1998). The self-organizing map. Neurocomputing, 21(May), 1-6.

Large, E. W., Almonte, F. V, \& Velasco, M. J. (2010). A canonical model for gradient frequency neural networks. Physica D, 239(12), 905-911. https://doi.org/10.1016/j.physd.2009.11.015

Laudanski, J., Coombes, S., Palmer, A. R., \& Sumner, C. J. (2010). Mode-Locked Spike Trains in Responses of Ventral Cochlear NucleusChopper and Onset Neurons to Periodic Stimuli. $J$ Neurophysiol, 103: 1226-. https://doi.org/doi:10.1152/jn.00070.2009

Meddis, R., \& Lowel P. O'Mard. (2006). Virtual pitch in a computational physiological model. $J$. Acoust. Soc, May, 3861-3870. https://doi.org/10.1121/1.2372595

Merzenich, M. M., Knight, P. L., \& Roth, G. L. (2018). Representation of cochlea within primary auditory cortex in the cat. Journal of Neurophysiology. https://doi.org/https://doi.org/10.1152/jn.1975.38.2.231

Merzenich, Michael M., Kaas, J. H., \& Roth, G. L. (1976). Auditory cortex in the grey squirrel: Tonotopic organization and architectonic fields. The Journal of Comparative Neurology, 387401.

Novick, A., \& Vaisnys, J. R. . (1964). Echolocation of Flying Insects by the Bat, Chilonycteris parnellii. Biological Bulletin, 127(3), 478-488. https://doi.org/doi.org/10.2307/1539249

Palakal, M. J., Murthy, U., Chittajallu, S. K., \& Wong, D. (1995). Tonotopic representation of auditory responses using self-organizing maps. Mathematical and Computer Modelling, 22(2), 7-21. https://doi.org/10.1016/0895-7177(95)00107-D

Palmer, A. R., \& Rees, A. (2010). The Oxford Handbook of Auditory Science: The Auditory Brain (A. R. Palmer \& A. Rees (eds.)). Oxford University Press. https://doi.org/10.1093/oxfordhb/9780199233281.001.0001

Penfield, B. Y. W. (1937). SOMATIC MOTOR AND SENSORY REPRESENTATION IN. Brain, 60:389-443.

Righetti, L., Buchli, J., \& Ijspeert, A. J. (2005). From Dynamic Hebbian Learning for Oscillators to Adaptive Central Pattern Generators. Proceedings of 3rd International Symposium on Adaptive Motion in Animals and Machines AMAM 2005, 1-7. https://doi.org/record/58529

Ritter, H., Martinez, T., \& Schulten, K. (1992). Neural computation and self-organizing maps: An introduction. Computation and neural systems series. https://doi.org/10.1057/jors.1994.32

Ruggero, M. A. (1992). Responses to sound of the basilar membrane of the mammalian cochlea. Current Opinion in Neurobiology, 2(4), 449-456. https://doi.org/10.1016/0959-4388(92)90179$\mathrm{O}$ 
889

890

891

892

893

894

895

896

897

898

899

900

901

902

903

904

905

906

907

908

909

910

911

912

913 coupled through modified power coupling which is identical as the single unit of the OTSOM model

914 under the special condition $\varepsilon \cong 0$ is described by eqns. 12,13 and 14 . The schematic of these networks

915 are shown in the fig. A1.

Schreiner, C. E., \& Sutter, L. (1992). Topography of Excitatory Bandwidth in Cat Primary Auditory Cortex : Single-Neuron Versus Multiple-Neuron Recordings. Journal of Neurophysiology, $68(5)$.

Simmons, J. A. (2012). Bats use a neuronally implemented computational acoustic model to form sonar images. Current Opinion in Neurobiology, 22(2), 311-319. https://doi.org/10.1016/j.conb.2012.02.007

Strogatz, S. H. (1994). Nonlinear dynamics and chaos. Addison Wesley.

Suga, N. (1990). Biosonar and neural computation in bats. Scientific American, 262(6), 60-68. https://doi.org/10.1038/scientificamerican0690-60

Suga, Nobuo, Yan, J., \& Zhang, Y. (1997). Cortical maps for hearing and egocentric selection for self-organization. Trends in Cognitive Sciences, 1(1), 13-20. https://doi.org/10.1016/s13646613(97)01002-4

Víctor M. Eguíluz, \& Ospeck, M. (2000). Essential Nonlinearities in Hearing. Physical Review Letters, June. https://doi.org/10.1103/PhysRevLett.84.5232

Wandell, B. A., Dumoulin, S. O., \& Brewer, A. A. (2007). Review Visual Field Maps in Human Cortex. Neuron, 1893, 366-383. https://doi.org/10.1016/j.neuron.2007.10.012

Yue, X., Robert, S., \& Ungerleider, L. G. (2020). Curvature processing in human visual cortical areas. NeuroImage, 222(August). https://doi.org/10.1016/j.neuroimage.2020.117295

\section{Appendix 1: A pair of Hopf oscillators coupled bilaterally and unilaterally through modifier power coupling.}

911 The dynamics of a pair of Hopf oscillators bilaterally coupled through modified power coupling is

912 described by the eqns. 6,7 and 8. Whereas the dynamics of a pair of Hopf oscillators unilaterally 


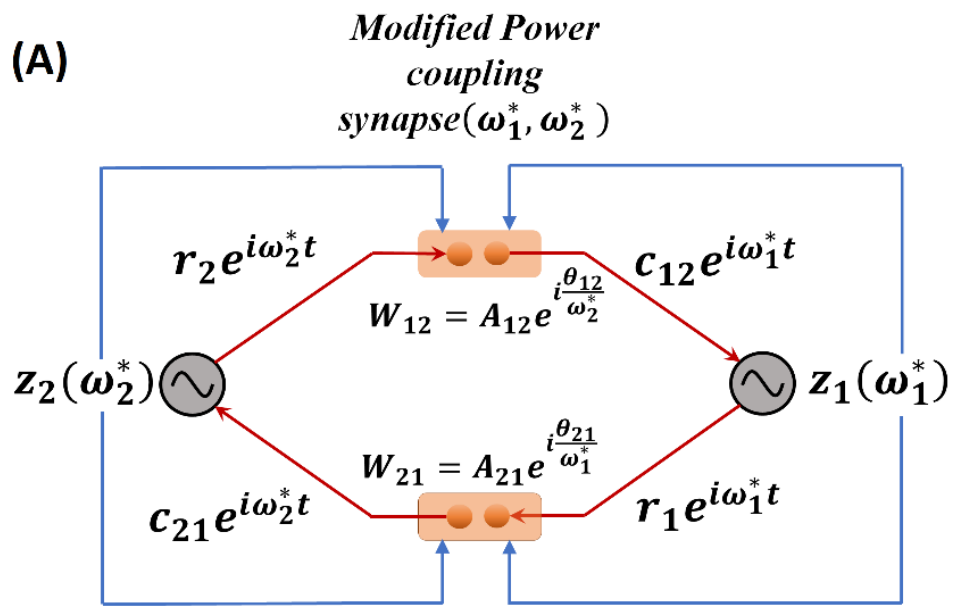

\section{(B)}

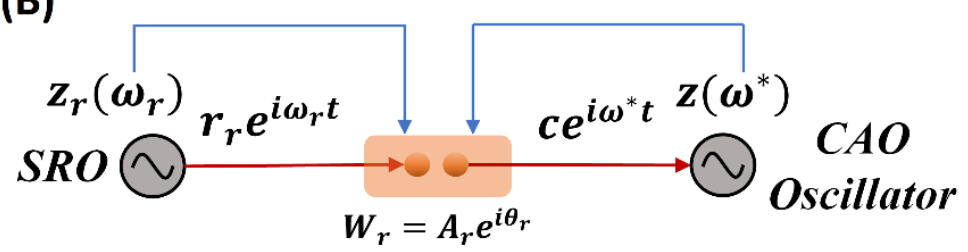

919 Network 1 (bilateral coupling): Initially both the oscillators will receive complex sinusoidal input

920 signal from the other oscillator through modified power coupling connection as $\omega_{1}^{*}$ and $\omega_{2}^{*}$ are 921 initialised to $\omega_{1}$ and $\omega_{2}$ respectively. As the Hopf oscillator keeps oscillating at its natural frequency, $922 \omega$, when it is perturbed by a complex sinusoidal signal with the same frequency, $\omega_{0}=\omega$, both of the 923 oscillators in the pair will continue to oscillate at a same frequency. So, $\omega_{1}^{*}$ and $\omega_{2}^{*}$ will remain as $\omega_{1}$

924 and $\omega_{2}$. Defining the new normalized phase difference as, $\lambda_{21}=\frac{\emptyset_{1}}{\omega_{1}^{*}}-\frac{\emptyset_{2}}{\omega_{2}^{*}}$, assuming at steady state

$925 \dot{\lambda}_{21}=\frac{\dot{\phi_{1}}}{\omega_{1}}-\frac{\dot{\phi_{2}}}{\omega_{2}}=0$, considering $\omega_{1}^{*}=\omega_{1}$ and $\omega_{2}^{*}=\omega_{2}$ all along. From equation $2 \mathrm{~b}$ and $3 \mathrm{~b}$,

926

$$
\begin{gathered}
1+\frac{A_{12} r_{2}^{\frac{\omega_{1}^{*}}{\omega_{2}^{*}}}}{\omega_{1} r_{1}} \sin \omega_{1}\left(\frac{\theta_{12}}{\omega_{1} \omega_{2}}+\frac{\emptyset_{2}}{\omega_{2}}-\frac{\emptyset_{1}}{\omega_{1}}\right)-1-\frac{A_{21} r_{1}^{\frac{\omega_{2}^{*}}{\omega_{1}^{*}}}}{\omega_{2} r_{2}} \sin \omega_{2}\left(\frac{\theta_{21}}{\omega_{1} \omega_{2}}+\frac{\emptyset_{1}}{\omega_{1}}-\frac{\emptyset_{2}}{\omega_{2}}\right)=0 \\
\frac{A_{12} r_{2} \frac{\omega_{1}}{\omega_{2}}}{\omega_{1} r_{1}} \sin \omega_{1}\left(\frac{\theta}{\omega_{1} \omega_{2}}+\frac{\emptyset_{2}}{\omega_{2}}-\frac{\emptyset_{1}}{\omega_{1}}\right)+\frac{A_{21} r_{1} \frac{\omega_{2}}{\omega_{1}}}{\omega_{2} r_{2}} \sin \omega_{2}\left(\frac{\theta}{\omega_{1} \omega_{2}}+\frac{\emptyset_{2}}{\omega_{2}}-\frac{\emptyset_{1}}{\omega_{1}}\right)=0
\end{gathered}
$$

928 As we have already assumed, $\theta_{12}=-\theta_{21}=\theta$, it is obvious that for some of the solutions of the above equation both the terms in the L.H.S of the equation would be 0 , in that case; 


$$
\frac{\theta}{\omega_{1} \omega_{2}}+\frac{\emptyset_{2}}{\omega_{2}}-\frac{\emptyset_{1}}{\omega_{1}}=n \pi
$$

$$
\lambda_{21}=n_{1} \pi+\frac{\theta}{\omega_{1} \omega_{2}}
$$$$
\sigma_{21}=\lambda_{21}-\frac{\theta}{\omega_{1} \omega_{2}}=\frac{\emptyset_{1}}{\omega_{1}}-\frac{\emptyset_{2}}{\omega_{2}}-\frac{\theta}{\omega_{1} \omega_{2}}=n_{1} \pi
$$

933 where, $n_{1}=-n$. So. The normalized phase difference has multiple solutions which depends on $\theta$ as

934 well as the natural frequency of both of the oscillators.

935 Network 2 (unilateral coupling): Initially the CAO oscillators will receive complex sinusoidal input 936 signal from the SRO through modified power coupling connection as $\omega^{*}$ is initialised to $\omega$. As the 937 Hopf oscillator keeps oscillating at its natural frequency, $\omega$, when it is perturbed by a complex 938 sinusoidal signal with the same frequency, $\omega_{0}=\omega$, CAO oscillator will continue to oscillate at a same 939 frequency. So, $\omega^{*}$ will remain as $\omega$. The normalized phase difference between CAO oscillator and SRO 940 will be:

$$
\lambda_{r}=\frac{\emptyset}{\omega^{*}}-\frac{\emptyset_{r}}{\omega_{r}}
$$

942 As $\omega^{*}$ will remain $\omega$ the normalised phase difference will be: $\lambda_{r}=\frac{\emptyset}{\omega}-\frac{\emptyset_{r}}{\omega_{r}}$. Assuming $\dot{\lambda_{r}}=0$ at steady943 state;

$$
\frac{\dot{\phi}}{\omega}-\frac{\dot{\phi}_{r}}{\omega_{r}}=0
$$

$$
A_{r} \frac{r_{r}^{\frac{\omega}{\omega_{r}}}}{r} \sin \left(\theta_{r}-\lambda_{r} \omega\right)=0
$$

946 The only solution of which is: $\lambda_{r}=\frac{\theta_{r}}{\omega}+n \pi$. So, the pair of unilaterally coupled Hopf oscillators 947 through modified power coupling will always synchronize with each other with a normalized phase 948 difference of $\frac{\theta_{r}}{\omega}+n \pi$.

949 Appendix 2: The steady state dynamical analysis of single unit as the CAO oscillator operates 950 under the entrainment regime of the input signal.

951 The approximate dynamics of eqns. $12,13,14$ considering $A_{r} \ll \varepsilon I_{0}$, when $\omega=\omega_{0}$ : 


$$
\tau_{\omega} \dot{\omega}^{*} \approx-\omega^{*}+\omega-\frac{\varepsilon I_{0}}{r} \sin \psi
$$

955 At steady state, $\dot{r}=0, \dot{\psi}=0$. From equation A2.2:

956

$$
\dot{\psi_{s s}} \approx-\frac{\varepsilon I_{0}}{r_{s s}} \sin \psi_{s s}=0
$$

$$
\text { i.e, } \quad \sin \psi_{s s}=0
$$

$$
\text { i.e, } \quad \psi_{s s}^{*}=n \pi
$$

959 The solutions $\psi_{s s}^{*}=(2 n+1) \pi$ are unstable whereas the solutions $\psi_{s s}^{*}=2 n \pi$ are stable. For the 960 stable solutions;

961

$$
\emptyset_{s s}-\omega_{0} t-\xi_{0}=2 \pi n
$$

962

$$
\delta_{s s}=2 \pi n+\xi_{0}
$$

963 i.e., the steady state phase offset of the CAO oscillator will be same as the phase offset of the input 964 signal. For $\psi_{s s}^{*}=2 n \pi$ solutions, equation A2.1 will become:

965

$$
\left(\mu-\beta_{1} r_{s S}^{2}\right) r_{S S}+\varepsilon I_{0} \approx 0
$$

966 From the last expression, the positive real solution, $r_{s S}$, of (A2.4) is derived with the help of

967 MathWorks equation solver:

$$
r_{S S}^{*}=\frac{\sqrt[3]{\frac{2}{3}} \mu}{\sqrt[3]{\sqrt{3} \sqrt{27 \varepsilon^{2} I_{0}{ }^{2} \beta_{1}{ }^{4}+4 \beta_{1}{ }^{3} \mu^{3}}+9 \varepsilon I_{0} \beta_{1}{ }^{2}}}-\frac{\sqrt[3]{\sqrt{3} \sqrt{27 \varepsilon^{2} I_{0}{ }^{2} \beta_{1}{ }^{4}+4 \beta_{1}{ }^{3} \mu^{3}}+9 \varepsilon I_{0} \beta_{1}{ }^{2}}}{\sqrt[3]{2} 3^{\frac{2}{3}} \beta_{1}}
$$

969 The solution of steady state magnitude of oscillation as found in eqn. A2.5 is numerically verified in

970 fig. A2.1. Whereas the fig. A2.2 elaborates the dependency of the entrainment width and the typical 971 transient time on the parameters $\mu, \beta_{1}$ and $\varepsilon I_{0}$. 


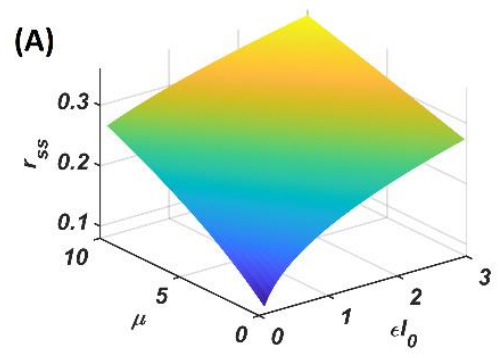

(D)

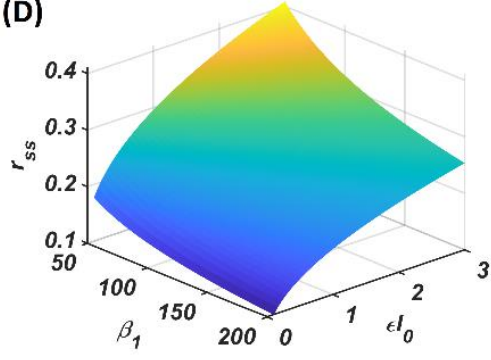

(G)

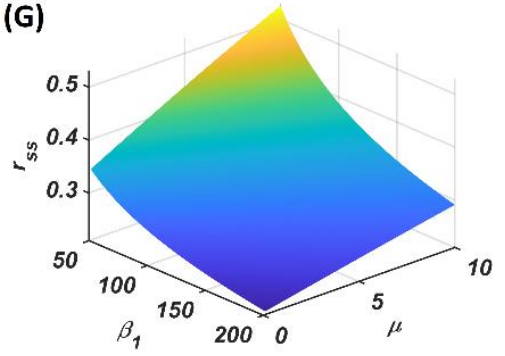

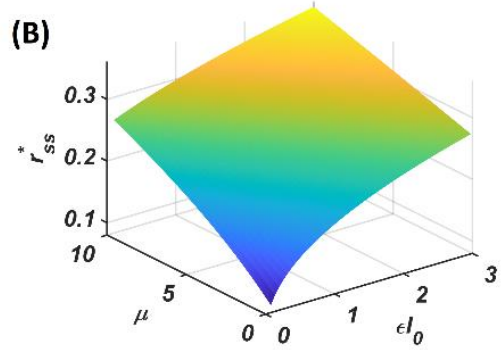

(E)

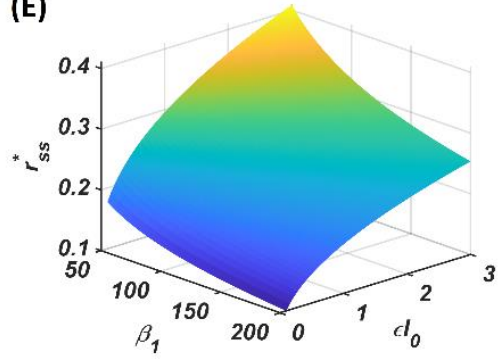

(H)

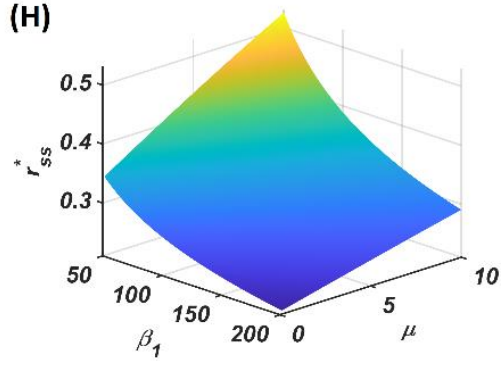

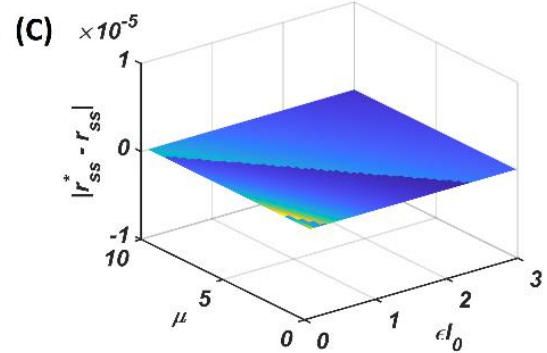

(F)

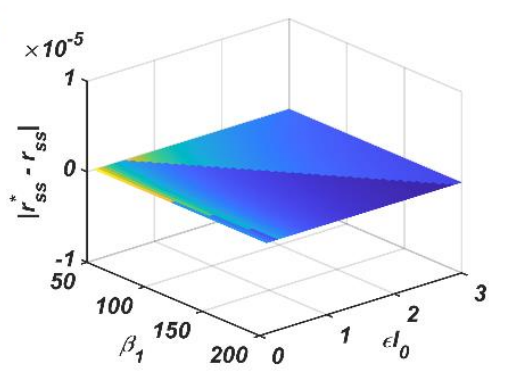

(I)

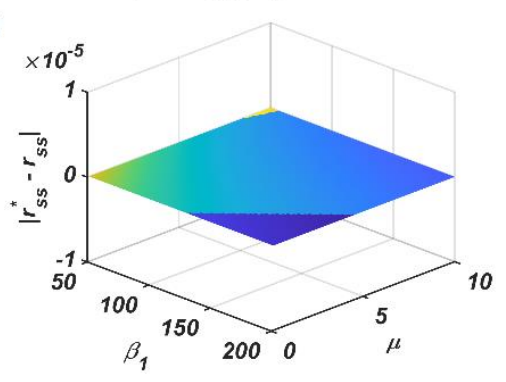

973 Figure A2.1: The first two columns (subplots A, D, G and B, E, H) proclaim that the dependency of 974 the solution $r_{s S}^{*}$ of the polynomial expression given by eqn.- A2.4 found by MathWorks polynomial 975 solver, given by the expression in eqn.- A2.5 on two of the three parameters $\mu, \beta_{1}$ and $\varepsilon I_{0}$ keeping the 976 remaining fixed at a certain intermediate value $\left(\varepsilon I_{0}=2, \beta_{1}=150, \mu=1\right)$ is identical to the steady 977 state magnitude of oscillation $\left(r_{S S}\right)$ deduced by simulating the transient dynamics of eqns.- A2.1 and 978 A2.2 with $\omega=\omega_{0}=2 \pi \times 60$. The error between the first two columns is plotted in the $3^{\text {rd }}$ column 979 (subplots $\mathbf{C}, \mathbf{F}, \mathbf{I}$ ). 
(A)

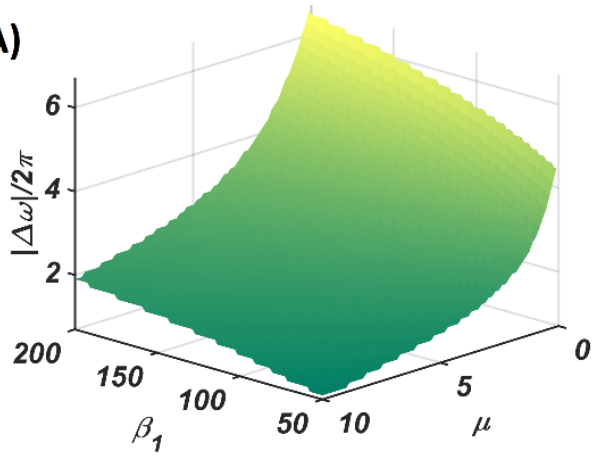

(C)

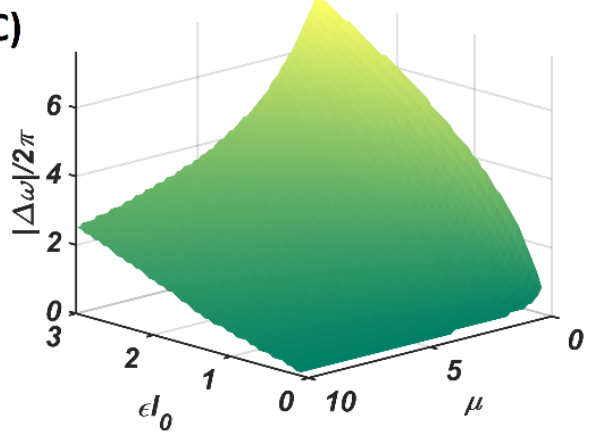

(E)

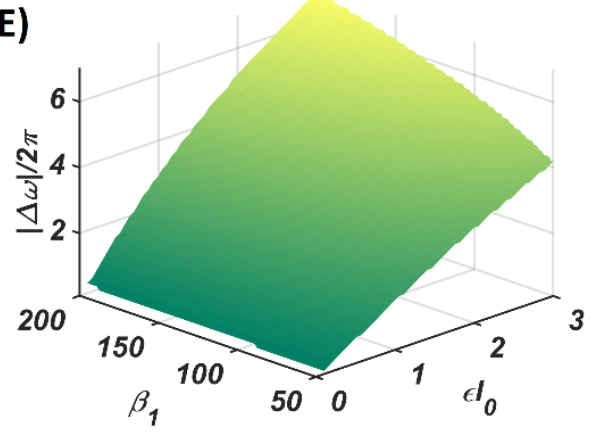

(B)
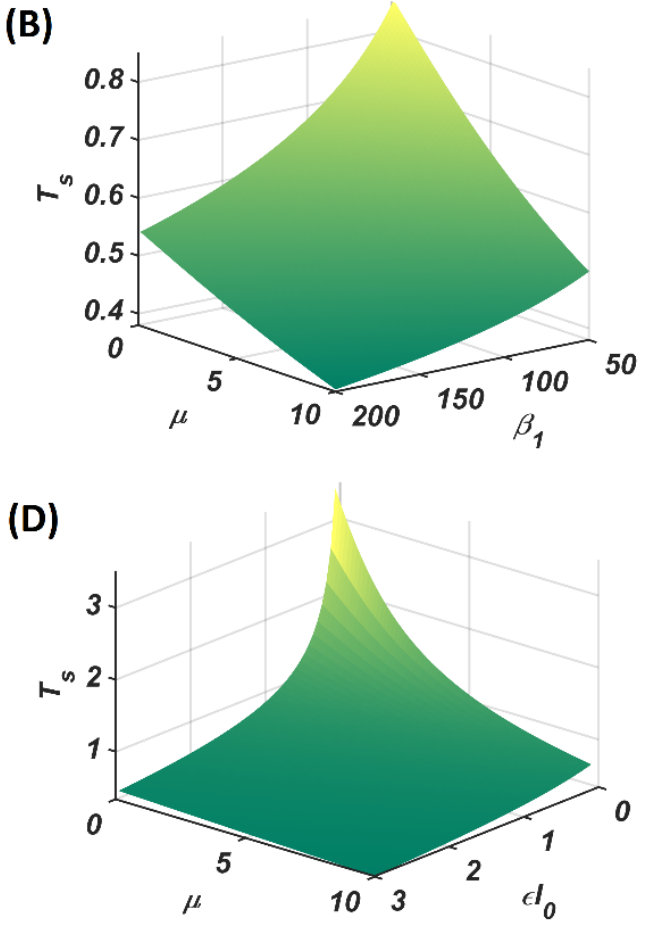

(F)

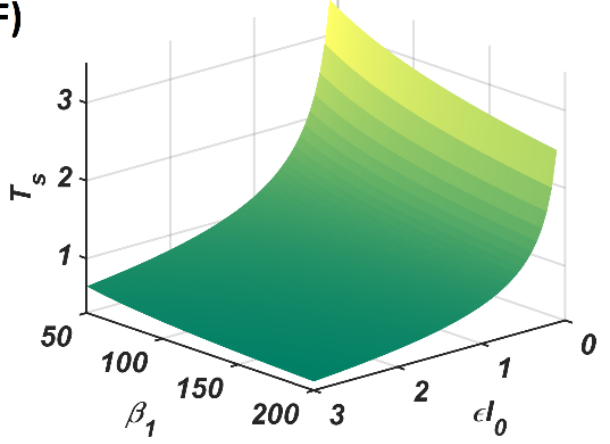

981 Figure A2.2: The subplots $\mathbf{A}, \mathbf{C}, \mathbf{E}$ delineates the dependency of the width of the entrainment regime $982(\Delta \omega)$ on two of the three parameters $\mu, \beta_{1}$ and $\varepsilon$ keeping the remaining fixed at a certain intermediate 983 value, similarly the remaining subplots delineates the dependency of $T_{s}$ (the time an individual 984 oscillator takes to attain steady-state under the influence of complex sinusoidal external input signal). 985 Parameter value: For $(\mathbf{A})$ and $(\mathbf{B}) \varepsilon I_{0}=2$, for $(\mathbf{C})$ and $(\mathbf{D}) \beta_{1}=150$ and for $(\mathbf{E})$ and $(\mathbf{F}) \mu=1, \omega$ is 986 kept fixed at $2 \pi \times 60 \mathrm{rad} / \mathrm{sec}$ while $\omega_{0}$ is varied from $2 \pi \times 45 \mathrm{rad} / \mathrm{sec}$ to $2 \pi \times 75 \mathrm{rad} / \mathrm{sec}$.

The approximate dynamics when the CAO oscillator is under the entrainment regime with $\omega \neq \omega_{0}$ :

$$
\dot{r} \approx\left(\mu-\beta_{1} r^{2}\right) r+\varepsilon I_{0} \cos \psi \quad A 2.6
$$

$$
\dot{\psi} \approx \Omega-\frac{\varepsilon I_{0}}{r} \sin \psi
$$

At steady state, equating eqn. A2.7 to zero; 


$$
\Omega-\frac{\varepsilon I_{0}}{r_{s s}} \sin \psi_{s s}=0
$$

993

$$
\sin \psi_{s s}=\frac{\Omega r_{s s}}{\varepsilon I_{0}}
$$

994 Substituting $\cos \psi_{s s}$ into eqn. A2.4 with the steady state assumption,

$$
\left(\mu-\beta_{1} r_{s S}{ }^{2}\right) r_{s S}+\varepsilon I_{0} \sqrt{1-\left(\frac{\Omega r_{S S}}{\varepsilon I_{0}}\right)^{2}}=0
$$

996

$$
\beta_{1}^{2} r_{s s}^{6}-2 \mu \beta_{1} r_{s s}^{4}+\left(\mu^{2}+\Omega^{2}\right) r_{s s}^{2}-\varepsilon^{2} I_{0}^{2}=0
$$

997 The solution of the expression can be drawn using MathWorks polynomial solver. The only positive 998 real solution of the equation is:

$999 \quad r_{S S}^{*}$

1000

$$
=\left(\frac{1}{3 \sqrt[3]{2} \beta_{1}^{2}} \sqrt[3]{27 \beta_{1}^{4} \varepsilon^{2} I_{0}{ }^{2}+18 \beta_{1}^{3} \Omega^{2} \mu+2 \beta_{1}^{3} \mu^{3}+\sqrt{4\left(3 \beta_{1}^{2} \Omega^{2}-\beta_{1}^{2} \mu^{2}\right)^{3}+\left(27 \beta_{1}^{4} \varepsilon^{2} I_{0}{ }^{2}+18 \beta_{1}^{3} \Omega^{2} \mu+2 \beta_{1}^{3} \mu^{3}\right)^{2}}}\right.
$$

$$
\left.-\frac{\sqrt[3]{2}\left(3 \beta_{1}^{2} \Omega^{2}-\beta_{1}^{2} \mu^{2}\right)}{3 \beta_{1}^{2} \sqrt[3]{27 \beta_{1}^{4} \varepsilon^{2} I_{0}{ }^{2}+18 \beta_{1}^{3} \Omega^{2} \mu+2 \beta_{1}^{3} \mu^{3}+\sqrt{4\left(3 \beta_{1}^{2} \Omega^{2}-\beta_{1}^{2} \mu^{2}\right)^{3}+\left(27 \beta_{1}^{4} \varepsilon^{2} I_{0}^{2}+18 \beta_{1}^{3} \Omega^{2} \mu+2 \beta_{1}^{3} \mu^{3}\right)^{2}}}}-\frac{2 \mu}{3 \beta_{1}}\right)^{\frac{1}{2}}
$$




$$
r_{S S}^{*}=\left(\frac{\sqrt[3]{27 \beta_{1} \varepsilon^{2} I_{0}{ }^{2}+18 \Omega^{2} \mu+2 \mu^{3}}}{3 \sqrt[3]{2} \beta_{1}} \sqrt[3]{1+\sqrt{\frac{4\left(3 \Omega^{2}-\mu^{2}\right)^{3}}{\left(27 \beta_{1} \varepsilon^{2} I_{0}{ }^{2}+18 \Omega^{2} \mu+2 \mu^{3}\right)^{2}}+1}}\right.
$$

$$
-\frac{\frac{\sqrt[3]{2}}{3}\left(3 \Omega^{2}-\mu^{2}\right)}{\sqrt[3]{27 \beta_{1}^{4} \varepsilon^{2} I_{0}^{2}+18 \beta_{1}^{3} \Omega^{2} \mu+2 \beta_{1}^{3} \mu^{3}}} \frac{1}{\sqrt[3]{1+\sqrt{\frac{4\left(3 \Omega^{2}-\mu^{2}\right)^{3}}{\left(27 \beta_{1} \varepsilon^{2} I_{0}^{2}+18 \Omega^{2} \mu+2 \mu^{3}\right)^{2}}}+1}}
$$

1004

$$
\left.-\frac{2 \mu}{3 \beta_{1}}\right)^{\frac{1}{2}}
$$

1005

1006

1007

1008

1009

1010

1011

1012

1013

1014

1015

$$
\dot{\omega}=-\eta_{\omega}\left(\operatorname{Re}\left(I_{e}(t)\right) \sin \emptyset-\operatorname{Im}\left(I_{e}(t)\right) \cos \emptyset\right)=f_{3}(r, \psi, \omega)
$$

1016 where, $I_{e}(t)=\varepsilon I(t)=\varepsilon I_{0} e^{i\left(\omega_{0} t+\xi_{0}\right)}$, the $\omega$ dynamics can be simplified as following: 
1017

1018

1019

1020

1021

1022

1023

1024

1025

1026

1027

1028

1029

1030

1031

1032

1033

1034

$$
\begin{gathered}
\dot{\omega}=-\eta_{\omega}\left(\operatorname{Re}\left(I_{e}(t)\right) \sin \emptyset-\operatorname{Im}\left(I_{e}(t)\right) \cos \emptyset\right) \\
=-\eta_{\omega}\left(\varepsilon I_{0} \cos \left(\omega_{0} t+\xi_{0}\right) \sin \emptyset-\varepsilon I_{0} \sin \left(\omega_{0} t+\xi_{0}\right) \cos \emptyset\right) \\
\dot{\omega}=-\eta_{\omega} \varepsilon I_{0} \sin \psi
\end{gathered}
$$

At steady state, $\omega_{s s}=-\eta_{\omega} \varepsilon I_{0} \sin \psi_{s s}=0$. The stable solution of which would be, $\psi_{s s}^{*}=2 n \pi$. Similarly, from eqn. A3.2,

$$
\dot{\psi_{s S}} \approx \Omega_{s S}-\frac{\varepsilon I_{0}}{r_{s s}} \sin \psi_{s s}=0
$$

i.e,

$$
\Omega_{s S}=0
$$

$$
\text { or, }
$$

$$
\omega_{s s}=\omega_{0}
$$

So, the steady state magnitude of oscillation will be the solution of the following expression as described in Appendix-2:

$$
\left(\mu-\beta_{1}{r_{S S}}^{2}\right) r_{s S}+\varepsilon I_{0} \approx 0
$$

In this scenario the phase offset of the CAO oscillator at steady state will be:

$$
\delta_{s S}=2 \pi n+\xi_{0}
$$

The corresponding Jacobian matrix for eqns. (A3.1-A3.3):

$$
\begin{gathered}
J=\left[\begin{array}{lll}
\frac{\partial f_{1}}{\partial r} & \frac{\partial f_{1}}{\partial \psi} & \frac{\partial f_{1}}{\partial \omega} \\
\frac{\partial f_{2}}{\partial r} & \frac{\partial f_{2}}{\partial \psi} & \frac{\partial f_{2}}{\partial \omega} \\
\frac{\partial f_{3}}{\partial r} & \frac{\partial f_{3}}{\partial \psi} & \frac{\partial f_{3}}{\partial \omega}
\end{array}\right] \\
J=\left[\begin{array}{ccc}
\mu-3 \beta_{1} r^{2} & -\varepsilon I_{0} \sin \psi & 0 \\
\frac{\varepsilon I_{0}}{r^{2}} \sin \psi & -\frac{\varepsilon I_{0}}{r} \cos \psi & 1 \\
0 & -\eta_{\omega} \varepsilon I_{0} \cos \psi & 0
\end{array}\right]
\end{gathered}
$$

The Jacobian matrix at the fixed point or the steady state solution:

$$
J=\left[\begin{array}{ccc}
\mu-3 \beta_{1} r_{s S}^{* 2} & 0 & 0 \\
0 & -\frac{\varepsilon I_{0}}{r_{s S}^{*}} & 1 \\
0 & -\eta_{\omega} \varepsilon I_{0} & 0
\end{array}\right]
$$

1035 Assuming $\beta_{1}<0$, the determinant $(\Delta)$ and the trace $(T)$ respectively: 


$$
\begin{gathered}
\Delta=\eta_{\omega} \varepsilon I_{0}\left(\mu-3 \beta_{1} r_{S S}^{* 2}\right) \\
T=\mu-3 \beta_{1} r_{S S}^{* 2}-\frac{\varepsilon I_{0}}{r_{S S}^{*}}
\end{gathered}
$$

1038 So, the solution $r_{S S}^{*}, \omega_{S S}^{*}=\omega_{0}$ and $\psi_{S S}^{*}=2 n \pi$ will be saddle point as $r_{S S}^{*}>\sqrt{\frac{\mu}{\beta_{1}}}$, which ensures $\mu-$ $3 \beta_{1} r_{S S}^{* 2}<0$, or $\Delta<0$.

\section{Appendix-4: Steady state dynamics of the transient phase of the second stage of training.}

1041 The dynamics of the single unit of the network during the transient phase of the $2^{\text {nd }}$ stage of training is 1042 defined by eqns. 12, 13, 14. The complex variable counterpart of eqns. 12 and 13 are:

$$
\begin{gathered}
\dot{z}=z\left(\mu+i \omega-\beta_{1}|z|^{2}\right)+A_{r} e^{i \theta_{r}} z_{r} \frac{\omega^{*}}{\omega_{r}}+\varepsilon I_{0} e^{i\left(\omega_{0} t+\xi_{0}\right)} \\
\text { or }, \quad \dot{z}=z\left(\mu+i \omega-\beta_{1}|z|^{2}\right)+I_{r}(t)+I_{e}(t) \\
\dot{z_{r}}=z_{r}\left(\mu_{r}+i \omega_{r}-\beta_{1 r}\left|z_{r}\right|^{2}\right) \quad A 4.2
\end{gathered}
$$

The complex activation of the reference oscillator at steady state,

$$
z_{r s s}=r_{r s s} e^{i \emptyset_{r s s}}=\sqrt{\frac{\mu_{r}}{\beta_{1 r}}} e^{i\left(\omega_{r} t+\emptyset_{r}(0)\right)}
$$

Assuming the special condition, $\Omega<\Delta \omega, \omega \neq \omega_{0}$ and $\epsilon_{\max }>\varepsilon I_{0}-A_{r}>\epsilon_{\min }$ i.e., the CAO oscillator is operating under the entrainment regime of the input signal (where $\Delta \omega$ depends on the $\mu$, $\beta_{1}, \varepsilon, F, \xi_{0}, \theta_{r}$ and $A_{r}$ ) with a visible amount of interference between $I_{e}(t)$ and $I_{r}(t)$, at steady state, the frequency of the CAO oscillator becomes the frequency of the input signal i.e., $\dot{\varnothing}$ or $\omega^{*}$ becomes equal to $\omega_{0}$. So, the steady state version of eqn. A4.1 can be written as:

$$
\dot{z}=z\left(\mu+i \omega-\beta_{1}|z|^{2}\right)+A_{r} e^{i \theta_{r}} \frac{\mu_{r} \frac{\omega_{0}}{2 \omega_{r}}}{\beta_{1 r}} e^{i\left(\omega_{0} t+\emptyset_{r}(0) \frac{\omega_{0}}{\omega_{r}}\right)}+\varepsilon I_{0} e^{i\left(\omega_{0} t+\xi_{0}\right)}
$$

$$
=z\left(\mu+i \omega-\beta_{1}|z|^{2}\right)+A_{r} \frac{\mu_{r} \frac{\omega_{0}}{\beta_{1 r}}}{\beta_{r}} e^{i\left(\omega_{0} t+\theta_{r}+\emptyset_{r}(0) \frac{\omega_{0}}{\omega_{r}}\right)}+\varepsilon I_{0} e^{i\left(\omega_{0} t+\xi_{0}\right)}
$$

$$
=z\left(\mu+i \omega-\beta_{1}|z|^{2}\right)+e^{i \omega_{0} t}\left(\varepsilon I_{0} e^{i \xi_{0}}+A_{r} \frac{\mu_{r} \frac{\omega_{0}}{\beta_{1 r}}}{2 \omega_{r}} e^{i\left(\theta_{r}+\emptyset_{r}(0) \frac{\omega_{0}}{\omega_{r}}\right)}\right)
$$




$$
\dot{z}=z\left(\mu+i \omega-\beta_{1}|z|^{2}\right)+\bar{F}_{n e t} e^{i \omega_{0} t}
$$

1057 where,

1058

$$
\bar{F}_{n e t}=\left(\varepsilon I_{0} e^{i \xi_{0}}+A_{r} \frac{\mu_{r} \frac{\omega_{0}}{2 \omega_{r}}}{\beta_{1 r}} e^{i\left(\theta_{r}+\emptyset_{r}(0) \frac{\omega_{0}}{\omega_{r}}\right)}\right)
$$

1059 is the phasor corresponding to the net input to the CAO oscillator at steady-state. The magnitude and 1060 the phase offset of $\bar{F}_{\text {net }}$ are:

1061

1062

$$
\begin{aligned}
& \left|\bar{F}_{n e t}\right|=\left(\left(\varepsilon I_{0} \cos \xi_{0}+A_{r}{\frac{\mu_{r}}{\beta_{1 r}}}^{\frac{\omega_{0}}{2 \omega_{r}}} \cos \left(\theta_{r}+\emptyset_{r}(0) \frac{\omega_{0}}{\omega_{r}}\right)\right)^{2}\right. \\
& \left.+\left(\varepsilon I_{0} \sin \xi_{0}+A_{r}{\frac{\mu_{r}}{\beta_{1 r}}}^{\frac{\omega_{0}}{2 \omega_{r}}} \sin \left(\theta_{r}+\emptyset_{r}(0) \frac{\omega_{0}}{\omega_{r}}\right)\right)^{2}\right)^{0.5} \\
& \arg \left(\bar{F}_{n e t}\right)=\tan ^{-1} \frac{\varepsilon I_{0} \sin \xi_{0}+A_{r} \frac{\mu_{r}}{\beta_{1 r}}{ }^{\frac{\omega_{0}}{2 \omega_{r}}} \sin \left(\theta_{r}+\emptyset_{r}(0) \frac{\omega_{0}}{\omega_{r}}\right)}{\varepsilon I_{0} \cos \xi_{0}+A_{r} \frac{\mu_{r} \frac{\omega_{0}}{\beta_{1 r}}}{2 \omega_{r}} \cos \left(\theta_{r}+\emptyset_{r}(0) \frac{\omega_{0}}{\omega_{r}}\right)}
\end{aligned}
$$

1064 The steady-state magnitude of oscillation is dependent on $\left|\bar{F}_{\text {net }}\right|$ whereas the phase offset of oscillation $\delta_{s S}=\left(\varnothing-\omega^{*} t\right)$ will be same as $\arg \left(\bar{F}_{n e t}\right)$ which is verified in fig. 10C.

1066 Appendix-5: Steady state dynamics of Hebbian plasticity phase of second stage of training.

1067 The approximate dynamics of the single unit at Hebbian plasticity phase of the $2^{\text {nd }}$ stage with the 1068 assumption $\varepsilon I_{0} \gg A_{r}$ :

$$
\dot{r} \approx r\left(\mu-\beta_{1} r^{2}\right)+\varepsilon I_{0} \cos \psi=f_{1}
$$

$$
\dot{\psi} \approx \Omega-\frac{\varepsilon I_{0}}{r} \sin \psi=f_{2}
$$

$$
\tau_{\omega} \dot{\omega}^{*} \approx-\omega^{*}+\omega-\frac{\varepsilon I_{0}}{r} \sin \psi
$$

$$
\dot{r}_{r}=\left(\mu_{r}-\beta_{1 r} r_{r}^{2}\right) r_{r}
$$

$$
\dot{\emptyset}_{r}=\omega_{r} \quad A 5.5
$$




$$
\dot{\theta_{r}}=-\frac{r r_{r}{ }^{\frac{\omega^{*}}{\omega_{r}}}}{\tau_{W} A_{r}} \sin \omega^{*}\left(\frac{\theta_{r}}{\omega^{*}}+\frac{\emptyset_{r}}{\omega_{r}}-\frac{\emptyset}{\omega^{*}}\right)=f_{3}
$$

1075

1076

1077

1078

1079

1080

1081

1082

1083

1084

1087

Considering the $\mathrm{CAO}$ oscillator is operating inside the entrainment regime, at steady state $\omega^{*}$ becomes $\omega_{0}$. From equation- $A 5.2$ we get;

$$
\sin \psi_{s S}=\frac{\Omega r_{s S}}{\varepsilon I_{0}}
$$

So, $\psi_{s S}^{*}=\sin ^{-1}\left(\frac{\Omega r_{S S}}{\varepsilon I_{0}}\right), \cos \psi_{s S}=\sqrt{1-\left(\frac{\Omega r_{S S}}{\varepsilon I_{0}}\right)^{2}}$ and the phase offset of the CAO oscillator at steadystate $\delta_{s S}^{*}=\xi_{0}+\sin ^{-1}\left(\frac{\Omega r_{S S}}{\varepsilon I_{0}}\right)$. The steady state dynamics of equation $A 5.6$ :

$$
\begin{gathered}
\dot{\theta_{r}}=-\frac{r r_{r} \frac{\omega^{*}}{\omega_{r}}}{\tau_{W} A_{r}} \sin \omega^{*}\left(\frac{\theta_{r}}{\omega^{*}}+\frac{\emptyset_{r}}{\omega_{r}}-\frac{\emptyset}{\omega^{*}}\right)=0 \\
\frac{r_{s s} r_{r s s} \frac{\omega_{0}}{\omega_{r}}}{\tau_{W} A_{r}} \sin \omega_{0}\left(\frac{\theta_{r s s}}{\omega_{0}}+\frac{\emptyset_{r s s}}{\omega_{r}}-\frac{\emptyset_{s s}}{\omega_{0}}\right)=0 \\
\sin \omega_{0}\left(\frac{\theta_{r s s}}{\omega_{0}}+\frac{\emptyset_{r s s}}{\omega_{r}}-\frac{\emptyset_{s s}}{\omega_{0}}\right)=0
\end{gathered}
$$

$$
\omega_{0}\left(\frac{\theta_{r s s}}{\omega_{0}}+\frac{\emptyset_{r s s}}{\omega_{r}}-\frac{\emptyset_{s S}}{\omega_{0}}\right)=n \pi
$$

At steady state, $\psi_{s s}=\sin ^{-1}\left(\frac{\Omega r_{S S}}{\varepsilon I_{0}}\right)$ or, $\emptyset_{s s}=\omega_{0} t+\xi_{0}+\sin ^{-1}\left(\frac{\Omega r_{S S}}{\varepsilon I_{0}}\right)$, and $\emptyset_{r s s}=\omega_{r} t$, assuming $\emptyset_{r}(0)=0$, substituting the values of $\emptyset_{r s s}$ and $\emptyset_{s s}$ into equation A4.8.

$$
\begin{array}{cc}
\theta_{r S S}+\omega_{0} t-\omega_{0} t-\xi_{0}-\sin ^{-1}\left(\frac{\Omega r_{S S}}{\varepsilon I_{0}}\right)=n \pi \\
\theta_{r s S}=n \pi+\xi_{0}+\sin ^{-1}\left(\frac{\Omega r_{S S}}{\varepsilon I_{0}}\right) & A 5.9
\end{array}
$$

Likewise, $r_{S S}^{*}$ would be the solution of the following expression:

$$
\begin{gathered}
\left(\mu+\beta_{1} r_{s s}{ }^{2}\right) r_{s s}+\varepsilon F \sqrt{1-\left(\frac{\Omega r_{s S}}{\varepsilon I_{0}}\right)^{2}}=0 \\
\beta_{1}{ }^{2} r_{s s}{ }^{6}+2 \mu \beta_{1} r_{s s}{ }^{4}+\left(\mu^{2}+\Omega^{2}\right) r_{s s}{ }^{2}-\varepsilon^{2} I_{0}{ }^{2}=0
\end{gathered}
$$


1094 Polar coordinate representation of the dynamics:

1095

$$
\dot{r}=\left(\mu-\beta_{1} r^{2}\right) r+A_{r} r_{r}^{\frac{\omega^{*}}{\omega_{r}}} \cos \omega^{*}\left(\frac{\emptyset}{\omega^{*}}-\frac{\theta_{r}}{\omega^{*}}-\frac{\emptyset_{r}}{\omega_{r}}\right)+\varepsilon I_{0} \cos \left(\omega_{0} t+\xi_{0}-\emptyset\right)
$$

1096

$$
\dot{\emptyset}=\omega-A_{r} \frac{r_{r}^{\frac{\omega^{*}}{\omega_{r}}}}{r} \sin \omega^{*}\left(\frac{\emptyset}{\omega^{*}}-\frac{\theta_{r}}{\omega^{*}}-\frac{\emptyset_{r}}{\omega_{r}}\right)+\frac{\varepsilon I_{0}}{r} \sin \left(\omega_{0} t+\xi_{0}-\emptyset\right)
$$

1098

$$
\dot{r_{r}}=r_{r}\left(\mu_{r}-\beta_{1 r} r_{r}^{2}\right)
$$

$$
\dot{\emptyset}_{r}=\omega_{r}
$$

1100

$$
\tau_{\omega} \dot{\omega}^{*}=-\omega^{*}+\omega-A_{r} \frac{r_{r}^{\frac{\omega^{*}}{\omega_{r}}}}{r} \sin \omega^{*}\left(\frac{\emptyset}{\omega^{*}}-\frac{\theta_{r}}{\omega^{*}}-\frac{\emptyset_{r}}{\omega_{r}}\right)+\frac{\varepsilon I_{0}}{r} \sin \left(\omega_{0} t+\xi_{0}-\emptyset\right)
$$

1101 A brief analysis of the steady state dynamics as follows:

Considering the CAO oscillator is operating inside the entrainment regime, at steady state $\omega^{*}$ becomes $\omega_{0}$. Writing eqns. A6.1 and A6.2 in terms of $\psi$ and $\Omega$.

$$
\tau_{W} \dot{\theta_{r}}=\frac{r r_{r}^{\frac{\omega^{*}}{\omega_{r}}}}{A_{r}} \sin \omega^{*}\left(\frac{\emptyset}{\omega^{*}}-\frac{\theta_{r}}{\omega^{*}}-\frac{\emptyset_{r}}{\omega_{r}}\right)
$$

$$
\begin{gathered}
\dot{r}=\left(\mu-\beta_{1} r^{2}\right) r+A_{r} r_{r}^{\frac{\omega^{*}}{\omega_{r}}} \cos \omega^{*}\left(\frac{\emptyset}{\omega^{*}}-\frac{\theta_{r}}{\omega^{*}}-\frac{\emptyset_{r}}{\omega_{r}}\right)+\varepsilon I_{0} \cos \psi \\
\dot{\psi}=\Omega-\frac{r_{r}^{\frac{\omega_{*}}{\omega_{r}}}}{r} \sin \omega^{*}\left(\frac{\emptyset}{\omega^{*}}-\frac{\theta_{r}}{\omega^{*}}-\frac{\emptyset_{r}}{\omega_{r}}\right)-\frac{\varepsilon I_{0}}{r} \sin \psi
\end{gathered}
$$

1106 In the entrainment regime, $\omega^{*}$ becomes $\omega_{0}$ at steady state. From eqn. A6.6 at steady state:

$$
\frac{r_{s S} r_{r S S}{ }^{\frac{\omega_{0}}{\omega_{r}}}}{A_{r}} \sin \omega_{0}\left(\frac{\emptyset}{\omega_{0}}-\frac{\theta_{r S S}}{\omega_{0}}-\frac{\emptyset_{r}}{\omega_{r}}\right)=0
$$

1108

$$
\sin \omega_{0}\left(\frac{\varnothing}{\omega_{0}}-\frac{\theta_{r S S}}{\omega_{0}}-\frac{\emptyset_{r}}{\omega_{r}}\right)=0 \text { or, } \cos \omega_{0}\left(\frac{\varnothing}{\omega_{0}}-\frac{\theta_{r S S}}{\omega_{0}}-\frac{\emptyset_{r}}{\omega_{r}}\right)=1
$$

$$
\frac{\theta_{r s s}}{\omega_{0}}=\frac{\emptyset}{\omega_{0}}-\frac{\emptyset_{r}}{\omega_{r}}
$$

From eqn. A6.2 at steady state; 


$$
\Omega-\frac{r_{r s s} \frac{\omega_{0}}{\omega_{r}}}{r_{s S}} \sin \omega_{0}\left(\frac{\emptyset}{\omega_{0}}-\frac{\theta_{r s s}}{\omega_{0}}-\frac{\emptyset_{r}}{\omega_{r}}\right)-\frac{\varepsilon I_{0}}{r_{s s}} \sin \psi_{s s}=0
$$

1112

$$
\begin{array}{r}
\psi_{s s}=\sin ^{-1} \frac{\Omega r_{s S}}{\varepsilon I_{0}} \\
\emptyset_{s s}=\omega_{0} t+\xi_{0}+\sin ^{-1} \frac{\Omega r_{s S}}{\varepsilon I_{0}}
\end{array}
$$

1114 From eqns. A6.8 and A6.9 assuming the reference oscillator was initialized at 0 phase, $\emptyset_{r}(0)=0$.

1115

$$
\begin{gathered}
\frac{\theta_{r s s}}{\omega_{0}}=\frac{\xi_{0}}{\omega_{0}}+\frac{1}{\omega_{0}} \sin ^{-1} \frac{\Omega r_{s s}}{\varepsilon I_{0}} \\
\boldsymbol{\theta}_{r s s}=\xi_{0}+\sin ^{-1} \frac{\boldsymbol{\Omega r _ { s s }}}{\varepsilon I_{0}}
\end{gathered}
$$

1117 From eqn. A6.1 we can find the steady state value of $r$ by finding the solution of the following equation;

$$
\left(\mu+\beta_{1} r_{s s}{ }^{2}\right) r_{s s}+A_{r} r_{r s s} \frac{\omega_{0}}{\omega_{r}}+\varepsilon I_{0} \sqrt{1-\left(\frac{\Omega r_{s S}}{\varepsilon I_{0}}\right)^{2}}=0
$$

1119

1120 